\title{
Self-Fulfilling Risk Panics
}

\author{
Philippe Bacchetta \\ University of Lausanne / CEPR
}

\section{Cedric Tille}

Graduate Institute for International and Development Studies / CEPR

\author{
Eric van Wincoop \\ University of Virginia / NBER
}

\begin{abstract}
The Recent crises have seen very large spikes in asset price risk without dramatic shifts in fundamentals. We propose an explanation for these risk panics based on self-fulfilling shifts in risk made possible by a negative link between the current asset price and risk about the future asset price. This link implies that risk about tomorrow's asset price depends on uncertainty about risk tomorrow. This dynamic mapping of risk into itself gives rise to the possibility of multiple equilibria and self-fulfilling shifts in risk. We show that this can generate risk panics. The impact of the panic is larger when the shift from a low to a high risk equilibrium takes place in an environment of weak fundamentals. The sharp increase in risk leads to a large drop in the asset price, decreased leverage and reduced market liquidity. We show that the model can account well for the developments during the recent financial crisis.
\end{abstract}

(C) The Authors.

All rights reserved. No part of this paper may be reproduced without the permission of the authors. 


\title{
Self-Fulfilling Risk Panics ${ }^{1}$
}

\author{
Philippe Bacchetta \\ University of Lausanne \\ CEPR
}

\author{
Cédric Tille \\ Graduate Institute, Geneva \\ CEPR
}

\author{
Eric van Wincoop \\ University of Virginia \\ NBER
}

June 28, 2010

\begin{abstract}
${ }^{1}$ We would like to thank Daniel Cohen, Luca Dedola, Stefan Gerlach, Paul Klein, Jaume Ventura, and audience members at the 2010 Bundesbank Annual Conference, the 2010 European Summer Symposium in International Macroeconomics, the ECB, NYFed, Dallas Fed, PSE, Cambridge, St. Andrews, Lausanne, Carlos III and the Hong Kong Monetary Authority for useful comments and discussions. We gratefully acknowledge financial support from the National Science Foundation (grant SES-0649442), the Hong Kong Institute for Monetary Research, the National Centre of Competence in Research "Financial Valuation and Risk Management" (NCCR FINRISK), and the Swiss Finance Institute.
\end{abstract}




\begin{abstract}
Recent crises have seen very large spikes in asset price risk without dramatic shifts in fundamentals. We propose an explanation for these risk panics based on selffulfilling shifts in risk made possible by a negative link between the current asset price and risk about the future asset price. This link implies that risk about tomorrow's asset price depends on uncertainty about risk tomorrow. This dynamic mapping of risk into itself gives rise to the possibility of multiple equilibria and self-fulfilling shifts in risk. We show that this can generate risk panics. The impact of the panic is larger when the shift from a low to a high risk equilibrium takes place in an environment of weak fundamentals. The sharp increase in risk leads to a large drop in the asset price, decreased leverage and reduced market liquidity. We show that the model can account well for the developments during the recent financial crisis.
\end{abstract}




\section{Introduction}

Sharp surges in risk are a prominent feature of financial panics, such as the turmoil in the Fall of 2008 or the 2010 Eurozone debt crisis. Volatility, as measured by the VIX index, more than quadrupled in the wake of the Lehman Brothers failure, and tripled during the debt crisis. While crises entail adverse fundamental news, these are hard-pressed to account for such large surges in risk. The precarious fiscal situation of Greece was long known. Similarly, while the 2008 panic was linked to large scale mortgage market losses, these were not suddenly discovered in the Fall of 2008 and had instead gradually built for at least a year prior to the panic. We offer a theory for such events, which we refer to as "risk panics", that focuses on sudden large self-fulfilling shifts in risk, as well as the volatility of risk. ${ }^{1}$

Our main contribution is to develop a theoretical foundation for such risk panics. Self-fulfilling shifts in risk can occur when the current equilibrium asset price depends on risk associated with the future asset price. ${ }^{2}$ Intuitively, higher risk reduces asset demand, which reduces the price. There is then a dynamic degree of freedom in the model. Risk is defined in terms of uncertainty about the asset price tomorrow. But the asset price tomorrow in turn depends on risk perceptions tomorrow. Therefore risk today depends on uncertainty about risk tomorrow. As risk does not just depend on uncertainty about future asset payoffs but also on future risk itself, self-fulfilling shifts in both risk and the volatility of risk are possible.

The possibility of self-fulfilling shifts in risk arises only when the current asset price depends on risk about the future asset price. This link is absent in the standard frictionless expected utility framework used in macroeconomics, where

\footnotetext{
${ }^{1}$ We do not wish to rule out the importance of fundamental shocks during the recent crises, but we are not aware of any model that would generate such a huge spike in risk in response to observed fundamental shocks. An alternative approach to ours is that of Caballero and Krishnamurthy (2008), who consider a model with Knightian uncertainty (i.e. inmeasurable risks). In that setup "new shocks" (e.g. the decision to let Lehman Brothers fail) can generate increased uncertainty as there is no history of events to measure probabilities. Another approach is found in Fostel and Geanakoplos (2008), where the economy can suddenly switch to a bad state with increased asset payoff risk. In our setup the increase in risk is entirely self-fulfilling and does not involve an exogenous increase in payoff risk.

${ }^{2}$ When we talk about "the asset price", we refer to a market portfolio of risky assets or stocks rather than the equity of a particular firm.
} 
only risk associated with future asset payoffs matters. The asset price then depends on the covariance between the stochastic discount factor and these payoffs. However, when introducing constraints on risk exposure, such as value-at-risk or margin constraints, the asset demand again depends on risk associated with future asset prices. Such constraints are natural in a world where highly leveraged financial institutions are subject to the possibility of default. A substantial literature introducing such constraints has developed in recent years. ${ }^{3}$

A simple way to model the impact of future risk and to illustrate the possibility of self-fulfilling risk shifts is to consider a mean-variance portfolio model. This can capture the impact of risk-based portfolio constraints, while avoiding their inherent complexity. ${ }^{4}$ Moreover, the mean-variance portfolio model has a long history in academics and is widely used in the financial industry. It has the important advantage of generating a simple relationship between future asset price risk and portfolio demand, which leads to a linear relationship between the asset price and future asset price risk in equilibrium.

We find that beyond a regular fundamental equilibrium there are equilibria in which risk and the volatility of risk fluctuate in an entirely self-fulfilling way. There is always a variable that is a coordination device for the self-fulfilling shifts in beliefs about risk. This can either be a variable extrinsic to the model or a macro fundamental that is part of the model. We refer to these as respectively sunspot and sunspot-like equilibria. ${ }^{5}$ In a sunspot-like equilibrium the fundamental variable plays a dual role. It affects the asset price both through its regular role as fundamental (e.g. through asset payoffs or wealth) and as a sunspot-like variable around which beliefs about risk are coordinated. ${ }^{6}$ Sunspot-like equilibria are con-

\footnotetext{
${ }^{3}$ Examples are Brunnermeier and Pedersen (2009), Danielsson, Shin and Zigrand (2009) and Gromb and Vayanos (2002). Danielsson et. al. (2009) find multiplicity in equilibrium risk through a feedback between asset prices and wealth. This mechanism is absent in our main analysis.

${ }^{4}$ Campbell et al. (2001) show that under some conditions the mean-variance and value-at-risk portfolio selections are the same.

${ }^{5}$ In the limiting case where fundamental uncertainty goes to zero, sunspot-like equilibria converge to pure sunspot equilibria.

${ }^{6}$ The term "sunspot-like" equilibria was first coined by Manuelli and Peck (1992). They write: "There are two ways that random fundamentals can influence economic outcomes. First, randomness affects resources which intrinsically affects prices and allocation. Second, the randomness can endogenously affect expectations or market psychology, thereby leading to excessive
} 
ceptually distinct from accelerator mechanisms where frictions in markets amplify the impact of a change in the fundamental variable. Those are pure fundamental equilibria.

Risk panics are closely related to the presence of sunspot-like equilibria. Apart from a pure fundamental equilibrium and sunspot-like equilibrium, the model also exhibits switching equilibria where there are exogenous shifts between a low-risk state and a high-risk state based on a Markov process. A panic is a switch from the low- to the high-risk state. During a panic, a macro variable suddenly becomes a focal point for self-fulfilling shifts in beliefs about risk. The panic is therefore not triggered by a change in the variable, but by the sudden self-fulfilling shift in beliefs about risk that is coordinated around this variable. The panic is larger when this variable is weak at the time of the shift (e.g. the net worth of leveraged institutions is low or the Greek debt is high).

Our theory is consistent with the two-stage pattern of the 2007-2008 crisis: while it started in mid-2007, a full scale financial panic did not hit until the Fall of 2008. We illustrate the self-fulfilling risk shift in a version of the model where investors (financial institutions) are hit by a negative wealth shock. We stress the dual role of the deteriorating net worth of financial institutions. First, it has a fundamental effect that can account for the initial stage of the crisis. It reduces liquidity in the market for risky assets, which raises the volatility of asset prices and lowers their levels. ${ }^{7}$ These effects are however relatively small. Second, it sets the stage for a large financial panic. This occurs when the low net worth suddenly becomes the focal point for a self-fulfilling increase in beliefs about risk. The lower the net worth is, the larger is the impact of the panic on asset prices, volatility, liquidity and leverage. ${ }^{8}$

volatility."

${ }^{7}$ The links between market liquidity, risk and financial leverage have received a lot of attention in recent contributions such as Adrian and Shin (2008), Brunnermeier and Pedersen (2009), Brunnermeier and Sannikov (2009), Gromb and Vayanos (2008), He and Krishnamurthy (2008a, b), Kyle and Xiong (2001) and Xiong (2001).

${ }^{8}$ It bears emphasizing that our focus in this paper is on the possibility of self-fulfilling risk shifts or risk panics. Every crisis has its own idiosyncratic aspects and the recent crisis obviously has many important features that go beyond the scope of this paper. For example, we abstract from aspects such as bank runs (through the repo market) and security complexity issues. We also make no attempt to account for the large losses in the securitized subprime mortgage market, 
The remainder of the paper is organized as follows. Section 2 shows how self-fulfilling shifts in risk naturally occur when the equilibrium price of an asset depends on the variance of the future asset price, regardless of the specifics of the model. In Section 3 we develop the possibility of sunspot and sunspot-like equilibria in a simple mean-variance portfolio model with stochastic asset payoffs (dividends). We consider both a simple model with a closed form solution and a more general one. In Section 4 we show that the model can also generate risk panics. Section 5 introduces wealth shocks, leading to an application to the 2007-2008 crisis in Section 6. Section 7 concludes.

\section{$2 \quad$ Self-Fulfilling Risk}

The key point of the paper is general and can be illustrated without relying on the specifics of a particular model. Consider a market where demand or supply depends not only on the current price but also on risk associated with the future price. The equilibrium price then depends on this risk. We write this in simple linear form as:

$$
Q_{t}=\lambda_{0}+\lambda_{1} \text { Risk }_{t}+\lambda_{2} E_{t} Q_{t+1}+\lambda_{3} y_{t}
$$

where $\operatorname{Risk}_{t}=\operatorname{var}_{t}\left(Q_{t+1}\right)$. Apart from risk, the price can depend on the expected price tomorrow and on a fundamental variable $y_{t}$ that exogenously shifts demand or supply. The expected future price naturally emerges in dynamic asset pricing models, but is not critical to the main point here (i.e. $\lambda_{2}$ could be zero). The key parameter for our point is $\lambda_{1}$, which relates the price to risk about the future price.

\subsection{Fundamental and sunspot equilibria}

Consider first a case where the price is not directly affected by the fundamental $y_{t}$, i.e. $\lambda_{3}=0$ in (1). It is immediate that there is an equilibrium, which we refer to as the fundamental equilibrium, where the price is constant:

$$
Q_{t}=\frac{\lambda_{0}}{1-\lambda_{2}}
$$

which we simply model as a negative wealth shock to leveraged investors. 
This is not the only equilibrium however. Consider equilibria where the asset price depends on a sunspot variable $S_{t}$ that does not enter (1):

$$
Q_{t}=\tilde{Q}-V S_{t}^{2}
$$

where $\tilde{Q}$ and $V$ are parameters. Assume that $S_{t}$ is persistent through an AR process:

$$
S_{t+1}=\rho S_{t}+\epsilon_{t+1}
$$

where $\rho \in\langle 0,1\rangle$ and the innovation $\epsilon_{t+1}$ has a symmetric distribution with mean zero and variance $\sigma^{2}$. We denote the variance of $\epsilon_{t+1}^{2}$ by $\omega^{2}$.

Equations (3) and (4) imply that:

$$
\begin{aligned}
& E_{t} Q_{t+1}=\tilde{Q}-V \rho^{2} S_{t}^{2}-V \sigma^{2} \\
& \operatorname{Risk}_{t}=\operatorname{var}_{t}\left(Q_{t+1}\right)=4 V^{2} \rho^{2} \sigma^{2} S_{t}^{2}+V^{2} \omega^{2}
\end{aligned}
$$

Note that risk is time-varying in a sunspot equilibrium where $V \neq 0$. Substituting (3), (5) and (6) into (1), solve for the parameters $\tilde{Q}$ and $V$ by equating respectively the constant terms and terms proportional in $S_{t}^{2}$ on the left and right hand side. The resulting system has two solutions. One is the fundamental equilibrium, with $V=0$ and $\tilde{Q}$ equal to (2), and the other is:

$$
\begin{aligned}
& V=-\frac{1-\lambda_{2} \rho^{2}}{4 \lambda_{1} \rho^{2} \sigma^{2}} \\
& \tilde{Q}=\frac{1}{1-\lambda_{2}}\left(\lambda_{0}+\lambda_{1} V^{2} \omega^{2}-\lambda_{2} V \sigma^{2}\right)
\end{aligned}
$$

The key parameter is clearly the coefficient on Risk $_{t}$ in (1). When risk does not affect the asset price $\left(\lambda_{1}=0\right)$, there is only the fundamental equilibrium. Otherwise the sunspot equilibrium also arises. The underlying intuition is most easily discussed when the expected future price does not enter (1), so that $\lambda_{2}=0$. In that case:

$$
Q_{t}=\lambda_{0}+\lambda_{1} \text { Risk }_{t}
$$

The same equation one period later tells us that the future asset price depends on future risk:

$$
Q_{t+1}=\lambda_{0}+\lambda_{1} \text { Risk }_{t+1}
$$


Taking the variance on both sides shows that current risk is linked to uncertainty about future risk:

$$
\operatorname{Risk}_{t}=\lambda_{1}^{2} \operatorname{var}_{t}\left(\operatorname{Risk}_{t+1}\right)
$$

Risk does not depend on uncertainty about future fundamental shocks, but instead on uncertainty about future risk itself. This dynamic mapping of risk into itself opens up the possibility for multiple equilibria. Clearly, zero risk is an equilibrium. But any process for Risk, unrelated to fundamentals, is an equilibrium as long as it satisfies (10). This process must clearly lead to joint shifts in risk and uncertainty about risk as they are proportional in (10). One process that satisfies (10) is described in (6), where Riskt is linear in $S_{t}^{2}$ and the sunspot follows an AR process. Uncertainty about future risk will then depend on $S_{t}^{2}$ as well because $\operatorname{var}_{t}\left(S_{t+1}^{2}\right)=4 \rho^{2} \sigma^{2} S_{t}^{2}+\omega^{2} .9$

\subsection{Sunspot-like equilibrium}

We have shown that when the fundamental $y_{t}$ does not enter (1), the model exhibits a fundamental and a pure sunspot equilibrium. The sunspot variable $S_{t}$ on which expectations of risk coordinate can be any variable. In particular, it can be the variable $y_{t}$. This corresponds to a situation where changes in a variable, such as the fiscal prospects of a country, affect the price in a market that is not linked to the country in any fundamental way.

Consider now the situation where $y_{t}$ has a fundamental impact, i.e. $\lambda_{3} \neq 0$ in (1). In addition to the fundamental equilibrium, there is a sunspot-like equilibrium where $y_{t}$ plays a dual role. It first has a fundamental role through its direct impact on the asset price in (1). It also has a sunspot role as the variable on which agents coordinate beliefs about risk.

To solve for the sunspot-like equilibrium, assume that $y_{t}$ follows the same autoregressive process as (4), and conjecture the following form of the asset price:

$$
Q_{t}=\tilde{Q}+v y_{t}-V y_{t}^{2}
$$

The parameters $\tilde{Q}, v$ and $V$ are solved analogously to that for the sunspot equilibrium. There are again two equilibria. The first is the fundamental equilibrium

\footnotetext{
${ }^{9}$ In fact, an alternative way to solve (9) is to simply conjecture $R i s k_{t}=\alpha+\beta S_{t}^{2}$ with $S_{t}$ an AR process. In that case (1) gives $Q_{t}$ and one can solve for $\alpha$ and $\beta$ by equating the conjectured process for Risk $k_{t}$ with that implied by the solution for the price.
} 
where $V=0, v=\lambda_{3} /\left(1-\lambda_{2} \rho\right)$ and $\tilde{Q}=\left(\lambda_{0}-\lambda_{1} v^{2} \sigma^{2}\right) /\left(1-\lambda_{2}\right)$. In the fundamental equilibrium the asset price depends linearly on $y_{t}$ (i.e. $V=0$ ), and risk is constant: Risk $_{t}=v^{2} \sigma^{2}$.

The other equilibrium is the sunspot-like equilibrium, where:

$$
\begin{aligned}
& V=-\frac{1-\lambda_{2} \rho^{2}}{4 \lambda_{1} \rho^{2} \sigma^{2}} \\
& v=-\frac{\lambda_{3} \rho}{1-\rho} \\
& \tilde{Q}=\frac{1}{1-\lambda_{2}}\left(\lambda_{0}+\lambda_{1}^{2} v^{2} \sigma^{2}+\lambda_{1} V^{2} \omega^{2}+\lambda_{2} V \sigma^{2}\right)
\end{aligned}
$$

This equilibrium converges to the pure sunspot equilibrium of the previous section in the limit where $\lambda_{3} \rightarrow 0$. In that pure sunspot equilibrium $y_{t}$ only plays a sunspot role $(v=0$ and $V \neq 0)$. As we raise $\lambda_{3}$ above $0, y_{t}$ takes on a dual role as a fundamental and a sunspot. But even as $\lambda_{3}$ becomes big, the sunspot role remains large. The coefficient $V$ in (11) is not a function of $\lambda_{3}$ at all and is identical to the pure sunspot case $(7)$. The term $V y_{t}^{2}$ in the equilibrium price therefore captures self-fulfilling shifts in risk coordinated around the variable $y_{t}$.

Although in a very different context, not involving self-fulfilling risk shifts, Manuelli and Peck (1992) and Spears, Srivastava and Woodford (1990) present models with sunspot-like equilibria. Spears, Srivastava and Woodford (1990) point out that "...a sharp distinction between "sunspot equilibria" and "non-sunspot equilibria" is of little interest in the case of economies subject to stochastic shocks to fundamentals." Indeed, as we raise $\lambda_{3}$ slightly above 0 , the sunspot-like equilibrium is technically no longer a pure sunspot equilibrium, but it is effectively indistinguishable.

\section{A Simple Mean-Variance Portfolio Choice Model}

We now show how the linkage between risk on the future asset price and the current price emerges in a simple mean-variance portfolio choice model. The model centers on the allocation of portfolios between risky equity and a risk-free bond. We first consider the case where the return on the bond is exogenous as this allows us to derive a closed-form solution for the fundamental and sunspot-like equilibria. We then endogeneize the interest rate in a full general equilibrium setup. 


\subsection{Model Description}

The model complexity is kept to a strict minimum. We consider an overlapping generation setup where investors are born with wealth $W_{I}$. They invest in equity and bonds and consume the return on their investment when old.

The bond pays an exogenous constant gross return $R$. This assumption, which is often made in the finance literature, allows us to derive a closed form solution. It implicitly assumes that there is a risk-free technology with a constant real return $R$ that is in infinite supply. This assumption is not crucial to our results and is relaxed in Section 3.5 below.

Equity consists of a claim on a tree with stochastic payoff. There are $K$ trees, each producing an exogenous stochastic output (dividend) $A_{t}$. Denoting the equity price by $Q_{t}$, the equity return from $t$ to $t+1$ is:

$$
R_{K, t+1}=\frac{A_{t+1}+Q_{t+1}}{Q_{t}}
$$

Agents face uncertainty both about the dividend and the future equity price. The dividend is equal to $\bar{A}\left(1+m S_{t}\right)$, where $S_{t}$ follows the process (4). $S_{t}$ is the only state variable in the model. When $m=0$ the dividend is a constant and $S_{t}$ becomes a pure sunspot. When $m>0, S_{t}$ has a fundamental impact on the equity payoff.

Investors born at time $t$ maximize a mean-variance utility over their portfolio return:

$$
E_{t} R_{t+1}^{p}-0.5 \gamma \operatorname{var}_{t}\left(R_{t+1}^{p}\right)
$$

where $\gamma$ measures risk aversion and the portfolio return is:

$$
R_{t+1}^{p}=\alpha_{t} R_{K, t+1}+\left(1-\alpha_{t}\right) R
$$

$\alpha_{t}$ denotes the portfolio share invested in equity. The gross return on equity and bonds are $R_{K, t+1}$ and $R$ respectively. The equity market clearing condition is

$$
\alpha_{t} W_{I}=Q_{t} K
$$

The OLG assumption is not critical to the results but simplifies the analysis in two ways. First, it avoids the well-known dynamic hedge term in the optimal portfolio that arises in multi-period portfolio problems. Second, the wealth level would be an additional state variable (in addition to $S_{t}$ ) if agents had infinite 
lives. We would then be unable to solve the model analytically or even represent the equilibria graphically. While we cannot get a closed form solution when the bond interest rate is endogenous, we can still represent the equilibria graphically as there is only one state variable. A shortcoming of the OLG assumption is that it prevents movements in asset prices from feeding back into the wealth of investors, a channel that can be important in a crisis. We introduce such a feedback effect in the Technical Appendix through a simple extension of the OLG setting, with a brief discussion in Section 6.3.

\subsection{Equilibrium Condition for Equity Price}

The maximization of (15) with respect to $\alpha_{t}$ gives the optimal portfolio share, which reflects the expected excess return on equity scaled by the variance of the equity return:

$$
\alpha_{t}=\frac{E_{t} R_{K, t+1}-R}{\operatorname{\gamma ar}_{t}\left(R_{K, t+1}\right)}
$$

Equation (17) does not restrict the portfolio share of equity to be lower than 100 percent. This share can exceed 100 percent when the equity return is not very risky, or when investors put little weight on risk. In that case the investors are leveraged, with long positions in equity and short positions in bonds.

Using (17), the market clearing condition (16) becomes:

$$
E_{t}\left(A_{t+1}+Q_{t+1}-R Q_{t}\right)=\frac{\gamma K}{W_{I}} \operatorname{var}_{t}\left(Q_{t+1}+A_{t+1}\right)
$$

Equation (18) equates the equilibrium expected excess payoff on equity to a risk premium that depends on the variance of the payoff $Q_{t+1}+A_{t+1}$. We use it to solve for the equilibrium asset price $Q_{t}$ as a function of the single state variable $S_{t}$.

\subsection{Sunspot Equilibria}

First consider the case where $m=0$, so that $S_{t}$ is a pure sunspot. In that case (18) can be written in the same form as (1) with $\lambda_{0}=\bar{A} / R, \lambda_{1}=-\gamma K /\left(R W_{I}\right)$ and $\lambda_{2}=1 / R$. We again get two equilibria. The first is the fundamental equilibrium where the asset price is constant:

$$
Q_{t}=\frac{\bar{A}}{R-1}
$$


The second is the sunspot equilibrium $Q_{t}=\tilde{Q}-V S_{t}^{2}$ where: ${ }^{10}$

$$
V=\frac{W_{I}}{K \gamma} \frac{R-\rho^{2}}{4 \rho^{2} \sigma^{2}}
$$

The intuition for the sunspot equilibrium is exactly the same as in Section 2.

An interesting point is that the impact of the sunspot on the equity price is larger when investors have a low risk aversion $\gamma$ or a large wealth $W_{I}$. As can be seen from (18), low risk aversion or large wealth reduce the risk premium and makes it less sensitive to changes in risk. It is precisely because agents respond less to risk (i.e. are less risk averse) that large self-fulfilling shifts in risk can more easily flourish. Paradoxically this implies that the asset price is more affected by such self-fulfilling shifts in risk.

\subsection{Sunspot-Like Equilibria}

Next consider the case where $m>0$, so that shocks to $S_{t}$ are also fundamental shocks to the asset payoff. We conjecture that the asset price is linear-quadratic in $S_{t}$ :

$$
Q_{t}=\tilde{Q}+v S_{t}-V S_{t}^{2}
$$

The only slight difference with the fundamental shocks to $y_{t}$ in Section 2 is that the risk is now given by $\operatorname{var}\left(Q_{t+1}+A_{t+1}\right)$ instead of $\operatorname{var}_{t}\left(Q_{t+1}\right)$. There are again two equilibria: a fundamental one and a sunspot-like one.

In the fundamental equilibrium we have $V=0$ and $v=m \rho /(R-\rho)$, and the asset price is:

$$
Q_{t}=\tilde{Q}+\frac{m \bar{A} \rho}{R-\rho} S_{t}
$$

Shocks have a bigger impact on the asset price when they are persistent. Asset price risk is constant.

In the sunspot equilibrium we have:

$$
V=\frac{W_{I}}{K \gamma} \frac{R-\rho^{2}}{4 \rho^{2} \sigma^{2}}
$$

\footnotetext{
${ }^{10} \mathrm{An}$ additional restriction to make sure that the asset price is always positive is that the distribution of $\epsilon_{t}$ is bounded. In that case $S_{t}$ is bounded as well. Since $(R-1) \tilde{Q}=\bar{A}-$ $V \sigma^{2}-\left(\gamma K / W_{I}\right) V^{2} \omega^{2}$, a sufficient condition for the asset price to always be positive is that $\bar{A}$ is sufficiently large.
} 


$$
v=-\frac{m \bar{A}}{1-\rho}
$$

As in Section 2, this sunspot-like equilibrium converges to the pure sunspot equilibrium as the fundamental shock vanishes to zero $(m \rightarrow 0)$. In addition, the coefficient on $S_{t}^{2}$ is again the same in the pure sunspot equilibrium (20) as in the sunspot-like equilibrium (23). $S_{t}$ therefore plays a double role of a macro fundamental that affects asset payoffs and as a sunspot that leads to self-fulfilling risk shifts.

\subsection{Full General Equilibrium}

Our analysis so far is not a full general equilibrium approach as we assume that there is an infinite supply of the risk-free bond at the rate of return $R$. In the remainder of the paper we relax this assumption and explicitly model the bond market equilibrium.

The bond market clearing condition equates the investors' demand for bonds to the supply. It is important that the supply of bonds be interest rate elastic. Otherwise, investors could not reallocate between stocks and bonds in equilibrium. The equity price would then be entirely pinned down by investors' wealth and there could be no sunspot or sunspot-like equilibria. There are many ways to introduce an interest rate elastic supply or demand schedule of bonds, for example by introducing interest elastic consumption/savings or investment decisions. We do so by introducing another set of agents, which we call households, who invest in bonds and a household technology detailed below.

There are overlapping generations of households born with wealth $W_{H}$. Households invest their endowment in bonds and a household technology, and consume the proceeds when old. Investing $K_{H, t+1}$ in the household technology at time $t$ yields a certain household production of $f\left(K_{H, t+1}\right)$ at $t+1$. The technology exhibits decreasing returns to scale, $f^{\prime}()>$.0 and $f^{\prime \prime}()<$.0 . Households therefore face no uncertainty. Households born at time $t$ maximize consumption at time $t+1$, which is equal to $f\left(K_{H, t+1}\right)+R_{t+1}\left(W_{H}-K_{H, t+1}\right)$, where $R_{t+1}$ is the interest rate on the bond. Consumption is maximized by equalizing the marginal return on the technology to the bond yield: $f^{\prime}\left(K_{H, t+1}\right)=R_{t+1}$.

For convenience we assume a simple quadratic form for household technology. 
The capital demand is then linear in the interest rate ${ }^{11}: K_{H, t+1}=\nu-\eta R_{t+1}$, and the demand for bonds by households is:

$$
W_{H}-K_{H, t+1}=W_{H}-\nu+\eta R_{t+1}
$$

Equation (25) can be positive, in which case households lend bonds to investors, or negative, in which case they borrow from investors.

The bond market clearing condition is:

$$
\left(1-\alpha_{t}\right) W_{I}+W_{H}-\nu+\eta R_{t+1}=0
$$

Using the equity market clearing condition (16), we rewrite this as

$$
Q_{t} K+\nu-\eta R_{t+1}=W
$$

where $W=W_{I}+W_{H}$ is the aggregate initial wealth. (26) gives a linear positive relationship between the equity price and the interest rate. A higher equity price raises the supply of equity. Clearing the equity market then requires investors to shift their portfolio towards equity and reduce their purchase of bonds (or borrow more from households). Bond market clearing then requires households to lower their borrowing (or increase their bond purchase), which they are induced to do through a higher interest rate. ${ }^{12}$

Using (26), the equity market clearing condition (18) becomes:

$$
E_{t}\left(A_{t+1}+Q_{t+1}-\frac{\nu-W}{\eta} Q_{t}-\frac{K}{\eta} Q_{t}^{2}\right)=\frac{\gamma K}{W_{I}} \operatorname{var}_{t}\left(Q_{t+1}+A_{t+1}\right)
$$

The equilibrium condition (27) only involves the equity price, which we again solve with the method of undetermined coefficients. We now no longer have an analytical solution because the time-varying interest rate leads to a non-linearity through the term $Q_{t}^{2}$ on the left hand side. We therefore adopt a numerical approximation method along the following lines (details are given in Appendix A). As is standard in the literature, we consider an approximation of the equilibrium asset price in logs:

$$
q_{t}=\tilde{q}+v S_{t}-V S_{t}^{2}
$$

\footnotetext{
${ }^{11}$ Specifically, we assume that $f\left(K_{H, t+1}\right)=\left[\nu K_{H, t+1}-0.5 K_{H, t+1}^{2}\right] / \eta$.

${ }^{12}$ There is a third market clearing condition, for goods, but we can drop it thanks to Walras' Law.
} 
We then take a quadratic approximation of $Q_{t}$ and $Q_{t+1}$ around $S_{t}=S_{t+1}=$ 0 , and use the result to compute the expectation and variance of $Q_{t+1}+A_{t+1}$. We substitute the resulting expressions into (27). We finally take a quadratic approximation around $S_{t}=0$, which gives a linear-quadratic expression in $S_{t}$ :

$$
Z_{0}+Z_{1} S_{t}+Z_{2} S_{t}^{2}=0
$$

where $Z_{0}, Z_{1}$, and $Z_{2}$ are functions of $\tilde{Q}=e^{\tilde{q}}, v$, and $V$. We solve for the value of these parameters by setting $Z_{0}=0, Z_{1}=0$, and $Z_{2}=0$.

While we are solving for three parameters, $\tilde{Q}, v$ and $V$, we can represent the equilibria graphically in a $(\tilde{Q}, v)$ space. Define $\tilde{V}=\tilde{Q} V$. In Appendix A we show that $Z_{0}=0$ implies

$$
\tilde{V}=\alpha_{1}+\alpha_{2} v^{2}
$$

where $\alpha_{1}$ and $\alpha_{2}$ are functions of $\tilde{Q}$. Substituting this into the expressions associated with $Z_{1}=0$ and $Z_{2}=0$ we obtain

$$
\begin{aligned}
& h_{1}+h_{2} v+h_{3} v^{2}+h_{4} v^{3}=0 \\
& g_{1}+g_{2} v+g_{3} v^{2}+g_{4} v^{3}+g_{5} v^{4}=0
\end{aligned}
$$

where $h_{i}$ and $g_{i}$ are functions of $\tilde{Q}$.

We solve numerically for the roots of the third and fourth order polynomials (31) and (32). The polynomials represent two schedules that map a given $\tilde{Q}$ into $v$, with possibly multiple solutions. We plot these two schedules in a $(\tilde{Q}, v)$ space with each intersection representing an equilibrium combination of $\tilde{Q}$ and $v$. $\tilde{V}$, and therefore $V$, then follow from (30).

For a given process for $S_{t}$ a typical parameterization gives 4 equilibria. This is illustrated in Figures 1 and 2 for respectively $m=0$ and $m=1$. Schedule (31) is represented by the solid line and (32) by the broken line. When $m=0$ the variable $S_{t}$ is a pure sunspot. Figure 1 shows that there is one fundamental equilibrium where $v=V=0$. The other three equilibria are all sunspot equilibria. The fact that for a given process for $S_{t}$ there are now three sunspot equilibria rather than the single sunspot equilibrium we found before is a result of the non-linearity generated by the time-varying interest rate.

In Figure 2, where $m=1, S_{t}$ is a fundamental that drives the asset payoffs. There are again 4 equilibria. Equilibrium 1 is a pure fundamental equilibrium. 
As we let $m \rightarrow 0$, it converges to Equilibrium 1 in Figure 1 where $v=V=$ 0 . The other three equilibria are all sunspot-like equilibria. As we let $m \rightarrow$ 0 , they converge to the corresponding sunspot equilibria in Figure 1. Figure 3 illustrates the convergence of the sunspot-like Equilibrium 2 of Figure 2 to the sunspot Equilibrium 2 of Figure 1 when $m$ goes to zero. It is remarkable that even when we get far away from $m=0, \tilde{Q}, v$ and $V$ change very little, especially in comparison to the near-zero levels of $v$ and $V$ in the fundamental equilibrium. This suggests that even when the fundamental role of $S_{t}$ is important, the impact of $S_{t}$ on the asset price is dominated by self-fulfilling shifts in risk.

\section{Risk Panics}

\subsection{Switching across states}

Risk panics can happen in equilibria that allow for a switch between low and high risk states. In the previous section the economy was either in a fundamental or sunspot-like equilibrium. We now consider an equilibrium that allows for switches between a low risk state (indexed by 1, akin to the fundamental equilibrium) and a high risk state (indexed by 2 , akin to the sunspot equilibrium). Switching occurs through an exogenous Markov process. The probability that we remain in a low risk state next period when we are in a low risk state today is $p_{1}>0.5$. Similarly, the probability that we remain in a high risk state next period when we are in a high risk state today is $p_{2}>0.5$.

Equilibria 1 and 2 in Figure 2 are the points to which the low and high risk states converge, respectively, in the limit where switching is not possible $\left(p_{1}=\right.$ $\left.p_{2} \rightarrow 1\right)$. When switching is possible, the low risk state becomes riskier than the pure fundamental equilibrium 1 in Figure 2. This is because there is now a possibility of switching to the high risk state, a switch that implies a significant drop in the equity price. Even when the probability of switching is low, the main source of uncertainty in the low risk state becomes the possibility of a jump to the high risk state rather than the pure fundamental uncertainty in Equilibrium 1 of Figure $2 .{ }^{13}$ Agents take the possibility of switching into account when forming

\footnotetext{
${ }^{13}$ This is similar to what is found in the "rare disaster" literature (e.g., Barro, 2006, Gabaix, 2009) where a small probability of a large disaster affects what happens in the no disaster periods.
} 
their expectations.

We conjecture that the log equity price in state $i$ is

$$
q_{i, t}=\tilde{q}_{i}+v_{i} S_{t}-V_{i} S_{t}^{2}
$$

As there are two such equations we solve for 6 unknown parameters (3 for each state). This is done by imposing equity market equilibrium as before, but separately for both states. We compute the expectation and variance of $Q_{t+1}$ taking into account that a switch to a different state is possible. The algebra is presented in Appendix B.

As an illustration, Figure 4 shows the values of $\tilde{Q}_{i}, v_{i}$ and $V_{i}$ in the low and high risk states for the case where $p_{1}=p_{2}$. As pointed out above, the two states correspond exactly to Equilibria 1 and 2 of Figure 2 when $p_{1}=p_{2}=1$. Switching equilibria only exist when the probability of remaining in the same state is high enough. But when $p_{1}=p_{2}$ is higher than this cutoff (sufficiently low probability of switching), the difference between the two states quickly becomes very big. A lower probability of switching particularly reduces risk in the low risk state (lower values of $v$ and $V$ ).

A risk panic is a switch from the low to the high risk state. Apart from the spike in risk, the panic also entails an increase in the volatility of risk, a sharp drop in the equity price and a shift out of equity (i.e. deleveraging when investors initially hold leveraged portfolios). We graphically illustrate these effects in Section 6 in an application to the 2008 financial crisis.

\subsection{Panics and fundamentals}

It is important to be clear both about the role that fundamentals do and do not play in a panic. First, a panic is not caused by a change in fundamentals. It happens for a given level of $S_{t}$. Second, the magnitude of the panic is larger the weaker the fundamental (the more negative $S_{t}$ ). Finally, once a panic occurs the asset price becomes much more sensitive to subsequent fluctuations in the fundamental. The market becomes on edge regarding any news about $S_{t}$.

Consider the first point: a panic does not result from a change in the fundamental. As can be seen from Figure 4, during the switch to the high risk state the coefficients $v$ and $V$ increase, generally by a large magnitude. This affects risk 
and the asset price for a given level of $S_{t}$. What changes is not $S_{t}$ itself but rather the role that it plays. As we switch to the high risk state, $S_{t}$ suddenly becomes a key variable around which agents coordinate their perceptions of risk. There is a sudden self-fulfilling increase in risk with the variable $S_{t}$ being the focal point for the change in risk perceptions. ${ }^{14}$

Notice that a risk panic is therefore conceptually distinct from financial accelerator models where the impact of shocks is magnified through financial constraints. While small shocks have a large effect in such models, the mechanism at work is a purely fundamental mechanism. Our framework instead puts the coordination of expectations center stage. During the panic asset prices and risk move sharply even though the state variable does not change.

Next consider the second point: the magnitude of the panic is larger the weaker the fundamental. To illustrate this point, consider the change in the equity price from the low to the high risk state. From (33) it follows that the change in the log equity price is

$$
q_{2, t}-q_{1, t}=\tilde{q}_{2}-\tilde{q}_{1}+\left(v_{2}-v_{1}\right) S_{t}-\left(V_{2}-V_{1}\right) S_{t}^{2}<0
$$

Since $v_{2}-v_{1}$ and $V_{2}-V_{1}$ are both positive (see Figure 4 ), the drop in the equity price is larger the more negative is $S_{t}$ (i.e. the weaker the fundamental). Consider for instance that $p_{1}=p_{2}=0.65$. In that case a panic lowers the equity price by only $13 \%$ when $S_{t}=0$, but by $65 \%$ when $S_{t}$ is two standard deviations below its unconditional mean of 0 .

In this light a large risk panic can also be viewed as a delayed amplification effect. Consider a deterioration of the fundamental (a drop in $S_{t}$ ) when the economy is in the low risk state. The shock lowers the equity price through the standard fundamental mechanism, but this impact is relatively small. The delayed amplification effect occurs if at some later date there is a switch to the high risk equilibrium. At that point, the sunspot role of $S_{t}$ suddenly surges. The impact of the panic on the asset price is much larger than the fundamental impact of $S_{t}$ in the first stage. We will further illustrate this point in Section 6 in the context of the recent financial crisis.

\footnotetext{
${ }^{14}$ Even in the low risk state $S_{t}$ plays to some extent a sunspot role if $p_{1}<1$. But this role is generally much stronger in the high risk state. In the low-risk state this role only reflects the possibility of switching to the high risk state.
} 
Finally consider the last role of the fundamental in a panic: once a panic occurs the asset price becomes much more sensitive to subsequent fluctuations in the fundamental. Once we switch to the high risk state, the fundamental $S_{t}$ becomes the focal point around which investors coordinate their beliefs about risk. This causes them to react strongly to any change in the variable. A further deterioration can lead to a significant further drop in the equity price. Conversely, an improvement in the fundamental becomes a significant stabilizing force. In the example above with $p_{1}=p_{2}=0.65$, the equity price drops from 100 to 35 during a panic when the fundamental is two standard deviations below its mean. But when the fundamental reverts to it mean, the equity price goes all the way back to 87 , even though we are still in the high risk state.

\section{$5 \quad$ Financial Shocks}

We now slightly modify our framework to show that the mechanism of self-fulfilling risk that we stress in this paper could explain various aspects of the recent financial crisis. We focus on financial shocks that redistribute wealth between households and investors. These shocks fit more closely the storyline of the 2007-2008 financial crisis where financial institutions experienced large negative shocks to their wealth (net worth) connected to mortgage market losses. For convenience, we abstract from aggregate shocks in our benchmark analysis, but we show that our results are robust to allowing for aggregate losses. Financial shocks that only affect the distribution of wealth impact the relative demand for bonds and equity as only investors are present in the equity market.

In addition to their closer link to the financial crisis, financial shocks are interesting as they give rise to another type of multiplicity through a circular relationship between risk and market liquidity. This type of multiplicity, that we call static multiplicity, has already been identified in the literature, although perhaps not in the context of a simple portfolio choice model. It is however distinct from the dynamic multiplicity that we identified in previous sections.

We make two changes relative to the model in Section 3.5. First, we assume that asset payoff shocks have no persistence $(\rho=0)$. This simplifies the analysis as the wealth of investors is then the only state variable. Second, we introduce 
shocks to the wealth of investors as follows:

$$
W_{I, t}=e^{-m \theta_{t}-0.5 m^{2} \theta_{t}^{2}} \bar{W}_{I}
$$

where

$$
\theta_{t+1}=\rho_{\theta} \theta_{t}+\epsilon_{t+1}^{\theta}
$$

and $\epsilon_{t+1}^{\theta}$ is a shock with mean zero and variance $\sigma_{\theta}^{2}$. Financial shocks only redistribute wealth and leave aggregate wealth unchanged: $W_{I, t}+W_{H, t}=W$. We assume that financial shocks $\epsilon_{t+1}^{\theta}$ and asset payoffs $A_{t+1}$ are uncorrelated. Equation (34) ensures that investors' wealth is linear in $\theta_{t}$ up to a quadratic approximation:

$W_{I, t}=\bar{W}_{I}\left(1-m \theta_{t}\right)$. A rise in $\theta_{t}$ implies a drop in the relative wealth of investors. The parameter $m$ allows us to vary the fundamental impact of wealth shocks. If $m=0, \theta_{t}$ does not affect wealth and becomes a pure sunspot.

The equity market clearing condition remains the same as (18), but now with time-varying wealth of investors:

$$
E_{t}\left(A_{t+1}+Q_{t+1}-R Q_{t}\right)=\frac{\gamma K}{W_{I, t}} \operatorname{var}_{t}\left(Q_{t+1}+A_{t+1}\right)
$$

The model is solved the same way as in Section 3.5, with a quadratic approximation of the market clearing condition around $\theta_{t}=0$. The details can be found in the Technical Appendix. The asset price is again a linear-quadratic function of the state variable:

$$
q_{t}=\tilde{q}-v \theta_{t}-V \theta_{t}^{2}
$$

We now examine more closely the two types of multiplicity that arise with financial shocks.

\subsection{Static Multiplicity: Interaction Between Risk and Liq- uidity}

The static multiplicity generated by financial shocks is linked to the concept of market liquidity. It is most clearly illustrated by assuming that the state variable $\theta_{t}$ shows no persistence $\left(\rho_{\theta}=0\right)$ so the model essentially becomes a static setting. Risk is then constant as the current state variable has no effect on the future asset price. 
Market liquidity is related to the price impact of asset demand shocks. Liquidity is low when shocks have a large impact on either the price or the expected excess payoff of the asset. The two are related as a larger change in the equilibrium expected excess payoff requires a larger adjustment of the current price. For our purposes it is convenient to define liquidity as the impact of wealth shifts on the expected excess payoff: ${ }^{15}$

$$
\frac{\partial E_{t}\left(A_{t+1}+Q_{t+1}-R_{t+1} Q_{t}\right)}{\partial W_{I, t}}
$$

A reduction in the wealth of investors (a higher $\theta_{t}$ ) lowers the demand for equity. The expected excess payoff on equity then needs to increase to bring investors back into the market. The more it increases, the more negative (37) is and the lower liquidity. Differentiating the equity market clearing condition (36), liquidity is equal to:

$$
-\frac{\gamma \operatorname{Kvar}_{t}\left(Q_{t+1}+A_{t+1}\right)}{W_{I, t}^{2}}
$$

Liquidity is low ((38) is more negative) when risk is high or wealth is low. High risk implies that equity is unattractive and investors take a small position in the equity market. With a limited exposure to the equity market, investors respond less to changes in the expected excess payoff on equity. Larger changes in the expected excess payoff are then necessary to clear the equity market, so liquidity is low. Similarly, lower wealth means that less money is on the line in the equity market. Larger changes in the expected excess payoff are then needed to clear the market. $^{16}$

The model implies a circular relationship between risk and liquidity. High risk implies that investors hold a small portfolio share in equity. The equity market is then thin and liquidity low. Low liquidity in turn implies a large price impact of asset demand shocks, so that risk is indeed high. This circular relationship leads to two equilibria for $\rho_{\theta}=0$. This is illustrated in Figure 5, which represents schedules (31) and (32). Apart from wealth shock parameters, the parameterization is the

\footnotetext{
${ }^{15}$ See Amihud et al. (2005) and Vayanos and Wang (2009) for surveys of various liquidity measures.

${ }^{16}$ When $\rho_{\theta}>0$ there will be an extra term in (38) associated with time-varying risk. It is equal to $\left[\gamma K / W_{I, t}\right]\left[\partial \operatorname{var}_{t}\left(Q_{t+1}+A_{t+1}\right) / \partial W_{I, t}\right]$. When a drop in wealth raises risk, it requires an even larger increase in the expected excess payoff to clear the market.
} 
same as used in Figures 1 and 2. Equilibrium 1 is the low risk equilibrium, where $v$ and $V$ are close to zero, while Equilibrium 2 is the high risk equilibrium.

The static multiplicity is closely related to multiple equilibria in limited participation models such as Pagano (1989), Allen and Gale (1994) and Jeanne and Rose (2002). ${ }^{17}$ In these models there are relatively few agents in the market in the high risk equilibrium. Liquidity is then low and risk high. The opposite is the case in the low risk equilibrium. Even though we do not allow agents to enter or exit the market, investors' exposure to equity is lower when risk is high.

While the static multiplicity has been recognized in the literature, its appeal is limited for two reasons. First, risk is constant within each equilibrium (low- and high risk), which is empirically not satisfactory. Second, the static multiplicity arises only for financial shocks, but not for shocks on asset payoffs for instance. With transitory asset payoff shocks, the only equilibrium is the fundamental one where the asset price and risk are constant.

\subsection{Dynamic Multiplicity: Sunspot and Sunspot-Like Equi- libria}

The dynamic multiplicity that arises when $\rho_{\theta}>0$ is the focus of the paper. It is illustrated in Figure 6, which uses the same parameterization as Figure 5 except that we set $\rho_{\theta}=0.4$. Panel A shows results for $m=2$ and Panel B for $m=0$. In both panels Equilibrium 1 is the fundamental equilibrium. The other equilibria in Panel A are all sunspot-like equilibria, which converge to the corresponding pure sunspot equilibria in Panel B when $m \rightarrow 0$. These equilibria again exhibit self-fulfilling shifts in risk, which are now coordinated around the variable $\theta_{t}$. The dynamic multiplicity can thus generate time-varying risk.

Even though the fundamental shock is now different from earlier sections, the sunspot-like equilibria in Panel A are quite similar to those in Figure 2 for the asset payoff shocks. The reason for this similarity is the dominance of the sunspot aspect of the variable. Any macro variable can play this role, irrespective of the particular role that is plays as a fundamental within the model.

\footnotetext{
${ }^{17}$ There are other examples of static multiplicity with different levels of risk in other contexts. See for example McCafferty and Driskill (1980) or Bacchetta and van Wincoop (2006).
} 


\section{Application to 2007-2008 Financial Crisis}

This section uses our setting to shed light on the 2007-2008 financial crisis. After presenting some basic financial data, we simulate the model and show that it generates an outcome qualitatively similar to what happened during the crisis. It should be emphasized that there are many important aspects of the recent crisis that are well beyond the scope of this paper. To the extent that our model is applicable in shedding light on the crisis, it is primarily in the context of the self-fulfilling shifts in risk perceptions that are the focus of this paper. We take the accumulating financial losses of leveraged investors as given (reflected in the financial shocks) and focus on the implications for the dynamics of risk, leverage, liquidity and asset prices.

\subsection{Dynamics of Risk, Leverage, Liquidity, and Asset Prices}

The crisis came in the form of a one-two punch. The first part is the relatively calm period from the beginning of 2007 until September 2008. The second part is the financial panic that started in September 2008. The panic peaked by the end of 2008 and it took several quarters for the situation to return to a more normal state. Using data for the United States, we focus on the following variables: (1) stock prices, (2) T-bill rate, (3) equity price risk, (4) volatility of risk, (5) net worth of leveraged institutions, (6) leverage, and (7) market liquidity. Stock prices are measured by the DJ U.S. total stock market index. Risk is measured as the CBOE SPX volatility VIX index. Volatility of risk is the standard deviation of the VIX index over the past 30 days. Net worth and leverage are based on U.S. brokers and dealers as reported by the Federal Reserve Flow of Funds. Market liquidity is difficult to measure in the data as it is a theoretical concept that does not have a straightforward empirical counterpart. We construct a measure similar to Amihud (2002) which, of different market liquidity measures, correlates the most with estimates of price impact computed using very high-frequency data (see Goyenko et al., 2009). Starting with individual stocks, we compute the average absolute daily stock price change over a month per dollar of daily trading volume. This is then averaged over 100 stocks from the S\&P index. ${ }^{18} \mathrm{~A}$ high value of our

\footnotetext{
${ }^{18}$ We are grateful to Giorgio Valente for providing us with the updated measure. Holding period returns and volumes are from Reuters Datastream. To deal with stationarity, in the spirit
} 
measure indicates low market liquidity. It is therefore a measure of illiquidity.

The dynamics of the variables during the crisis are illustrated in Figure 7. The vertical line represents the collapse of Lehman Brothers on September 15, 2008, which we consider to be the start of the financial panic. After a modest decline in stock prices and a small increase in risk during the tranquil period of the crisis, stock prices suddenly crashed and risk spiked in September 2008. The volatility of risk also shot up, while it showed no trend in the first stage. A flight to quality lowered the T-bill rate to near zero. Net worth gradually declined after mid 2007 until the third quarter of 2008, to quickly recover after the crisis. Financial leverage first rose significantly during the tranquil period, and then fell sharply during the panic stage. Finally, liquidity fell modestly during the tranquil part of the crisis, followed by a sharp drop in liquidity during the panic and then a return back to normal by mid-2009.

\subsection{Model Simulation}

We illustrate the dynamics of the variables in the model, and relate them to the recent crisis, using the two-state switching equilibrium as described in Section 4. The parameters are shown at the bottom of Figure 8. The main results are robust to the precise parameter values chosen, as discussed below. We set $p_{1}=0.95$ and $p_{2}=0.7$. This ensures that the high risk state occurs much less frequently than the low risk state, as the economy spends only $14 \%$ of the time in the high risk state. Panics of a large magnitude are even less frequent because they require not only a switch to the high risk state but also a very weak fundamental.

The parameterization is chosen to make sure that investors are substantially leveraged. Investors' initial equity holdings are four times their net worth (wealth), and are financed by borrowing from households through bonds. High leverage is characteristic of most financial institutions. We therefore also refer to the investors as leveraged financial institutions. ${ }^{19}$

of Acharya and Pedersen (2005) the illiquidity measure is multiplied by the ratio of the aggregate volume for all stocks in the sample at the end of a month to the same aggregate volume at the beginning of the sample.

${ }^{19}$ While leverage is less than seen in the data for brokers and dealers, our investors should be seen as an aggregate of all investors. A possible refinement is to consider two sets of investors with different degrees risk aversion. A first group of "long" investors with a relatively high risk aversion 
We simulate the model over 16 periods, which we interpret as quarters. We do no make any attempt to match the process of financial losses in the data, but instead illustrate the drivers of the model through a simple step function for $\theta_{t}$, along with a simple switching between low and high risk states. The dynamics of $\theta_{t}$ are illustrated through the wealth of investors, which follows the same path, in the first chart of Figure 8. The economy is initially in a low risk state with $\theta_{t}$ at its unconditional mean of zero. $\theta_{t}$ rises from 0 to 0.3 in period 2 , which we can think of as Q1 2007 when the losses of leveraged institutions on mortgage securities became apparent, leading to a reduction of their the wealth. This situation lasts until period 8, which we can think of as Q3 2008, where the economy switches to the high risk state. It stays in that situation until period 11 (Q2 2009) when $\theta_{t}$ falls back to zero thanks, for example, to a recapitalization of leveraged institutions. The economy reverts back to the low risk state in period 14 (Q1 2010).

These dates are not meant to match the exact length of the panic or the period of financial weakness of leveraged financial institutions. Our focus is instead to highlight the separate roles of the financial health of leveraged institutions and the specific risk state. This is done by considering all possible combinations of financial health (normal versus bad) and the state (low risk, high risk) in order to evaluate the specific contribution of both elements.

The simulation is presented in Figure 8. Periods during which $\theta_{t}$ changes are marked by vertical dotted lines, while the shaded area denotes the time spent in the high risk state. The wealth of investors follows the overall pattern seen in the data for brokers and dealers in Figure 7, although the deterioration of the net worth of financial institutions was obviously more gradual in the data. The other panels show the paths of the equity price, risk, the volatility of risk, interest rate, leverage and illiquidity. The stock price (normalized at 100 initially) and gross interest rate are $Q_{t}$ and $R_{t+1}$. Risk is measured as the standard deviation of $Q_{t+1} / Q_{t}$, taking into account the possibility of switching to another state. The volatility of risk is

would not be leveraged, while the second group with low risk aversion would be the "leveraged" investors. This would connect somewhat closer to reality, but would not fundamentally change any of the results. The basic market clearing equation (18) would be similar with two groups of investors. The only difference is that the ratio $W_{I, t} / \gamma$ would be replaced by a risk-aversion weighted wealth $\left(W_{L, t} / \gamma_{L}+W_{N L, t} / \gamma_{N L}\right)$, where the subscripts $L$ and $N L$ stand for respectively leveraged and non-leveraged investors. 
the standard deviation at time $t$ of our risk measure at $t+1 .^{20}$ Leverage is equal to the share of equity in investors' portfolio, $\alpha_{t}$. Finally, illiquidity is measured as the absolute value of the derivative of the log equity price with respect to $\theta_{t}$. This connects well to the Amihud measure used in the data, which is also meant to capture the price impact of shocks. Results are very similar when illiquidity is defined as the impact of $\theta_{t}$ on the expected excess payoff, as in Section 5.1.

During the tranquil part of the crisis the shift in wealth away from leveraged financial institutions reduces demand for equity and therefore its price. It also leads to a decline in liquidity (see Section 5.1), which increases risk and reduces the equity price further. Nonetheless Figure 8 shows that these effects are all quite modest. The only large change is leverage, which almost doubles. While the small increase in risk reduces leverage, this is more than offset by an increase in the expected excess return due to the lower equity price. $^{21}$

The second stage of the crisis, when the economy shifts into the high-risk stage, is characterized by a surge in risk and its volatility. This prompts a sharp reduction in the equity price and leverage. The drop in leverage in turn dries up liquidity in the equity market. The switch to bonds leads to a sharp drop in the interest rate.

An important message from Figure 8 is that a large surge in risk requires two ingredients, either one of which alone is not sufficient. First, there needs to be a selffulfilling risk panic (switch to the high risk state). Second, the fundamental around which the market perceptions of risk coalesce (net worth of leveraged institutions) must be weak. A deterioration of the macro fundamental alone is not enough to generate a surge in risk. Even though the net worth of leveraged institutions drops by more than $50 \%$ during the first stage of the crisis, risk remains relatively modest. A switch to the high risk state by itself is not enough either. Risk is restored slightly below its pre-panic level in period 11, when we are still in the high risk state but the leveraged institutions are recapitalized.

While the simple exercise we have conducted here is not meant to match precise data, the overall pattern in these variables is broadly in line with the data in Figure

\footnotetext{
${ }^{20}$ In computing the volatility of risk, we assume that we remain in the same state the next period. This makes it more consistent with the data, where it is measured as the volatility of risk over the past 30 days, which usually captures volatility within the same state.

${ }^{21}$ The model does not account for the drop in the interest rate prior to the panic as that is largely related to monetary policy.
} 
7. During the pre-panic state of the crisis the impact on the equity price, risk and liquidity is quite modest in both the data and the model. The substantial increase in financial leverage during this period is also consistent with that in the model. Then, during the switch to the panic state the model accounts for the sharp drop in the equity price, financial leverage, and market liquidity and the sharp increase in risk.

The volatility of risk also behaves similarly to that in the data. It surges together with risk during the panic and later on declines with the fall in risk itself. This joint behavior of risk and the volatility of risk is a critical element of the model, as discussed in Section 2.1. Risk spikes in the model only because future risk becomes more uncertain.

\subsection{Sensitivity Analysis}

Self-fulfilling shifts in risk occur as long as the asset price is negatively affected by risk about the future asset price. One might therefore expect the findings in the simulation above to apply much more broadly than for the particular model assumptions and parameterization underlying Figure 8. We confirm this through a variety of sensitivity analysis that we summarize here, with the details given in the Technical Appendix.

We first check that the results in the simulation exercise presented in Figure 8 are robust to alternative parameter values. This is done by halving and doubling most parameters. The results remain qualitatively intact for all alternative parametrizations. In particular, a risk panic leads to a sharp increase in risk and the volatility of risk, and a large decrease in the equity price, market liquidity and leverage. The precise magnitudes are certainly sensitive to parameterization. In particular, the size of the risk panic is larger the smaller $\eta, \rho, \gamma$, and $m$ and the larger $\nu-W$.

Second, we assess how the specifics of the model affect the results. We have already seen that the nature of the fundamental around which risk panics are coordinated is not critical to the results, as shocks to asset payoffs also lead to multiple equilibria and risk panics. Another modeling aspect is the assumption that financial shocks redistribute wealth between investors and households, with no aggregate loss. We consider an alternative where the wealth loss for investors 
is not offset by a gain for households and find that the results remain very similar. Lastly, we abstracted from any feedback of the asset price to wealth. We include this aspect in our OLG setting by assuming that some of the endowment when born consists of trees. This amplifies the risk panic. For example, when $29 \%$ of the wealth is subject to asset price shocks (in the low risk state at $\theta_{t}=0$ ), we find that the feedback effect from the asset price to wealth increases the magnitude of risk panics, with risk spiking from $26 \%$ during the tranquil part of the crisis all the way to $129 \%$ at the height of the panic.

Finally, we check the robustness with respect to the approximation in the solution method. This is done by considering a cubic approximation of the market clearing condition instead of a quadratic one. The simulation results are not substantially affected, providing confidence that the precision of the approximation method is not critical to the results.

\section{Conclusion}

Motivated by several recent crises that have shown very large spikes in risk without correspondingly large shifts in fundamentals, we develop a theory for self-fulfilling shifts in risk. These shifts can occur when the asset price depends negatively on the perceived risk of the future asset price. Risk associated with tomorrow's asset

price then depends on uncertainty about risk tomorrow. This dynamic mapping of risk into itself gives rise to the possibility of self-fulfilling shifts in risk.

Although a risk panic occurs without any change in fundamentals, it has a larger impact the weaker the macro fundamental on which agents coordinate their perceptions of risk at the time of the panic. The sharp increase in risk and accompanying volatility of risk in turn give rise to a large drop in the asset price, decreased leverage and reduced market liquidity. The model can generate a twostage crisis, where a deteriorating fundamental at first generates a modest impact on risk, asset prices and market liquidity, followed later on by a panic stage with much larger movements as the weak macro fundamental suddenly becomes the focal point for a self-fulfilling spike in risk. This matches the developments during the 2007-2008 financial crisis.

Our findings open up several directions for future research. First, the equilibria that we have identified can be found in any model where the actions of agents 
depend on the risk of an endogenous variable. While we have focused on asset markets, the same may be the case for example in goods and labor markets. The issue is also not limited to prices. We could replace $Q$ with any other variable that depends on risk associated with its future level. This could for example be output. It is well-known that reduced uncertainty about the future economic environment is good for business today (e.g. see Bloom, 2009).

Another direction for future research is to consider multiple assets. In our entire analysis there is only one risky asset. This should therefore be interpreted as the market portfolio of risky assets, which could be a country-wide or even a global equity index. A natural question is what the implications are for stocks of individual firms. Closely related, in an open economy context one would like to know whether all countries will be affected by a risk panic or whether it could be contained to a limited number of countries. This question relates to the widely discussed issue of financial contagion and is analyzed in Bacchetta and van Wincoop (2010).

A final direction for further research pertains to financial crises. We have kept the model as simple as possible to focus on the role of self-fulfilling risk shifts. A natural question is how this interacts with other elements that we have ignored for convenience. A non-exhaustive list includes financial constraints on leveraged institutions (borrowing constraints, value at risk constraints), the possibility of default and associated bank runs, and the interaction between the financial crisis and real economic activity. Moreover, a crucial issue is the policy recommendation that arises from our analysis. In Bacchetta et al. (2010) we examine the role of leveraged institutions in the context of our model. We find that, despite their stabilizing role in normal times, less risk averse leveraged institutions increase the magnitude of risk panics. We conclude that a policy making financial institutions more risk averse, or more prudent, could substantially reduce volatility. 


\section{Appendix}

\section{A Numerical Solution of Model in Section 3.5}

In this Appendix we describe the solution of the equilibria in the version of the model in Section 3.5. We take a quadratic approximation of the market clearing condition around $S_{t}=0$. Before doing so, we first need to compute the expectation and variance of $Q_{t+1}+A_{t+1}$. From the conjecture (28) we have

$$
Q_{t}=\tilde{Q} e^{v S_{t}-V S_{t}^{2}}
$$

where $\tilde{Q}=e^{\tilde{q}}$. A quadratic approximation around $S_{t}=0$ gives

$$
Q_{t}=\tilde{Q}\left(1+v S_{t}+\left(-V+0.5 v^{2}\right) S_{t}^{2}\right)
$$

For consistency we now also model the asset payoff in logs: $\ln \left(A_{t}\right)=\ln (\bar{A})+m S_{t}-$ $0.5 \mathrm{~m}^{2} S_{t}^{2}$. This specification implies that a quadratic approximation of $A_{t}$ around $S_{t}=0$ is $A_{t}=\bar{A}\left(1+m S_{t}\right)$. Using these quadratic approximations of $Q_{t}$ and $A_{t}$ at $t+1$ and then substituting $S_{t+1}=\rho S_{t}+\epsilon_{t+1}$ gives

$$
\begin{aligned}
E_{t}\left(Q_{t+1}+A_{t+1}\right)= & \tilde{Q}\left(1+v \rho S_{t}+\left(-V+0.5 v^{2}\right)\left(\rho^{2} S_{t}^{2}+\sigma^{2}\right)\right)+ \\
& \bar{A}+m \bar{A} \rho S_{t} \\
\operatorname{var}\left(Q_{t+1}+A_{t+1}\right)= & \tilde{Q}^{2}\left(v+\left(-V+0.5 v^{2}\right) 2 \rho S_{t}\right)^{2} \sigma^{2}+ \\
& m^{2} \bar{A}^{2} \sigma^{2}+2 \tilde{Q} \bar{A}\left(v+\left(-V+0.5 v^{2}\right) 2 \rho S_{t}\right) \sigma^{2} m
\end{aligned}
$$

Here we have simplified slightly by adopting approximation $\epsilon_{t+1}^{2}=\sigma^{2}$ or $\operatorname{var}\left(\epsilon_{t+1}^{2}\right)=$ 0 . This holds exactly in a simple distribution where $\epsilon_{t}$ can only take on the values $-\sigma$ and $+\sigma$. More generally, it is frequently adopted as a continuous time approximation. Under a normal distribution the variance of $\epsilon_{t+1}^{2}$ is $2 \sigma^{4}$, which is a small fourth-order term. Dropping this small term makes it easier to represent the equilibria graphically.

Substituting these results into the market clearing condition (27) and taking a quadratic approximation around $S_{t}=0$ gives an equation of the form (29). Setting the coefficients $Z_{0}, Z_{1}$ and $Z_{2}$ equal to zero, we obtain respectively

$$
\begin{aligned}
& \bar{W}\left(\bar{A}+\tilde{Q}+\tilde{Q}\left(-V+0.5 v^{2}\right) \sigma^{2}-\frac{1}{\eta}(\nu-W) \tilde{Q}-\frac{1}{\eta} K \tilde{Q}^{2}\right)= \\
& K m^{2} \bar{A}^{2} \sigma^{2}+\tilde{Q}^{2} K v^{2} \sigma^{2}+K 2 \tilde{Q} \bar{A} v \sigma^{2} m
\end{aligned}
$$




$$
\begin{aligned}
& \bar{W} \tilde{Q} v\left(\rho-\frac{1}{\eta}(\nu-W)-\frac{1}{\eta} 2 K \tilde{Q}\right)+m \bar{W} \bar{A} \rho=4 K \tilde{Q}^{2} v\left(-V+0.5 v^{2}\right) \rho \sigma^{2}+ \\
& 4 K \tilde{Q} \bar{A}\left(-V+0.5 v^{2}\right) \rho \sigma^{2} m \\
& \bar{W}\left[\left(-V+0.5 v^{2}\right) \rho^{2}-\frac{1}{\eta}(\nu-W)\left(-V+0.5 v^{2}\right)-\frac{1}{\eta} 2 K \tilde{Q}\left(-V+v^{2}\right)\right]= \\
& 4 K \tilde{Q}\left(-V+0.5 v^{2}\right)^{2} \rho^{2} \sigma^{2}
\end{aligned}
$$

Here we define $\bar{W}=W_{I} / \gamma$.

The strategy is as follows. For a given value of $\tilde{Q}$ we first solve $\tilde{Q} V$ from (43) as a quadratic function of $v$. We substitute the result in (44) and (45). This gives respectively a third and fourth order polynomial in $v$ that needs to be solved numerically. This leads to two schedules that map $\tilde{Q}$ into $v$ (possibly multiple values of $v$ ) that can be graphed. Equilibria are the points where these schedules intersect.

From (43) we can solve

$$
\tilde{Q} V=\alpha_{1}+\alpha_{2} v+\alpha_{3} v^{2}
$$

where

$$
\begin{aligned}
\alpha_{1} & =\frac{1}{\sigma^{2}}\left(\bar{A}+\tilde{Q}-\frac{1}{\eta}(\nu-W) \tilde{Q}-\frac{1}{\eta} K \tilde{Q}^{2}\right)-\frac{K \bar{A}^{2} m^{2}}{\bar{W}} \\
\alpha_{2} & =-\frac{2 K \tilde{Q} \bar{A} m}{\bar{W}} \\
\alpha_{3} & =0.5 \tilde{Q}-\frac{\tilde{Q}^{2} K}{\bar{W}}
\end{aligned}
$$

From (44) we have

$$
\beta_{1}+\beta_{2} v+\beta_{3} v^{2}+\beta_{4} v^{3}+\beta_{5}[\tilde{Q} V]+\beta_{6}[\tilde{Q} V] v=0
$$

where

$$
\begin{aligned}
& \beta_{1}=\bar{W} \bar{A} \rho m \\
& \beta_{2}=\bar{W} \tilde{Q}\left(\rho-\frac{1}{\eta}(\nu-W)-\frac{1}{\eta} 2 \tilde{Q} K\right) \\
& \beta_{3}=-2 K \tilde{Q} \bar{A} \rho \sigma^{2} m \\
& \beta_{4}=-2 K \tilde{Q}^{2} \rho \sigma^{2} \\
& \beta_{5}=4 K \bar{A} \rho \sigma^{2} m \\
& \beta_{6}=4 K \tilde{Q} \rho \sigma^{2}
\end{aligned}
$$


Finally, (45) can be written as

$$
\lambda_{1} v+\lambda_{2} v^{2}+\lambda_{3} v^{4}+\lambda_{4}[\tilde{Q} V]+\lambda_{5}[\tilde{Q} V]^{2}+\lambda_{6}[\tilde{Q} V] v^{2}=0
$$

where

$$
\begin{aligned}
& \lambda_{1}=0 \\
& \lambda_{2}=0.5 \bar{W} \tilde{Q} \rho^{2}-\frac{1}{\eta} 0.5 \bar{W} \tilde{Q}(\nu-W)-\frac{1}{\eta} 2 \bar{W} K \tilde{Q}^{2} \\
& \lambda_{3}=-K \tilde{Q}^{2} \rho^{2} \sigma^{2} \\
& \lambda_{4}=-\bar{W}\left(\rho^{2}-\frac{1}{\eta}(\nu-W)-\frac{1}{\eta} 2 \tilde{Q} K\right) \\
& \lambda_{5}=-4 K \rho^{2} \sigma^{2} \\
& \lambda_{6}=4 K \tilde{Q} \rho^{2} \sigma^{2}
\end{aligned}
$$

Substituting (46) into (50), we have

$$
h_{1}+h_{2} v+h_{3} v^{2}+h_{4} v^{3}=0
$$

where

$$
\begin{aligned}
& h_{1}=\beta_{1}+\beta_{5} \alpha_{1} \\
& h_{2}=\beta_{2}+\beta_{6} \alpha_{1}+\beta_{5} \alpha_{2} \\
& h_{3}=\beta_{3}+\beta_{5} \alpha_{3}+\beta_{6} \alpha_{2} \\
& h_{4}=\beta_{4}+\beta_{6} \alpha_{3}
\end{aligned}
$$

Substituting (46) into (57), we have

$$
g_{1}+g_{2} v+g_{3} v^{2}+g_{4} v^{3}+g_{5} v^{4}=0
$$

where

$$
\begin{aligned}
& g_{1}=\lambda_{4} \alpha_{1}+\lambda_{5} \alpha_{1}^{2} \\
& g_{2}=\lambda_{1}+\lambda_{4} \alpha_{2}+2 \lambda_{5} \alpha_{1} \alpha_{2} \\
& g_{3}=\lambda_{2}+\lambda_{4} \alpha_{3}+2 \lambda_{5} \alpha_{1} \alpha_{3}+\lambda_{6} \alpha_{1}+\lambda_{5} \alpha_{2}^{2} \\
& g_{4}=2 \lambda_{5} \alpha_{2} \alpha_{3}+\lambda_{6} \alpha_{2} \\
& g_{5}=\lambda_{3}+\lambda_{5} \alpha_{3}^{2}+\lambda_{6} \alpha_{3}
\end{aligned}
$$


Equations (64) and (69) are third and fourth order polynomials that we solve numerically. The solutions map $\tilde{Q}$ into $v$. There may be multiple solutions (multiple $v$ for a given $\tilde{Q}$ ). We then plot these two schedules in a space with $v$ on the vertical axis and $\tilde{Q}$ on the horizontal axis, as in Figures 1-2. There is an equilibrium when the two schedules intersect. The precise equilibria can be found by solving (43)-(45) numerically in Gauss as a fixed point problem in $v, V$ and $\tilde{Q}$. We choose starting values that are close to the equilibria found through visual inspection of where the two schedules intersect. Visual inspection gives approximate values for $\tilde{Q}$ and $v$. The corresponding value for $V$ follows from (46).

\section{B Solving the Switching Equilibria}

We now consider the equilibria in Section 4 of the paper where we allow for a switch between a low and high risk state. $p_{1}\left(p_{2}\right)$ is the probability that next period we will be in the low (high) risk state when this period we are in the low (high) risk state. The log equity prices in the low and high risk states are

$$
\begin{aligned}
& q_{t}^{\text {low risk }}=\tilde{q}_{1}+v_{1} S_{t}-V_{1} S_{t}^{2} \\
& q_{t}^{\text {high risk }}=\tilde{q}_{2}+v_{2} S_{t}-V_{2} S_{t}^{2}
\end{aligned}
$$

Assume that currently we are in the low risk state at time $t$. Analogous to (41), the expectation of $Q_{t+1}+A_{t+1}$, conditional on being in a low risk state in $t+1$, is

$$
E_{t+1}\left(Q_{t+1}+A_{t+1} \mid t+1 \text { is low }\right)=a_{1, \text { low }}+a_{2, \text { low }} S_{t}+a_{3, \text { low }} S_{t}^{2}
$$

where $a_{1, \text { low }}=\tilde{Q}_{1}\left(1+\omega_{1} \sigma^{2}\right)+\bar{A}, a_{2, \text { low }}=\tilde{Q}_{1} v_{1} \rho+m \bar{A} \rho, a_{3, \text { low }}=\tilde{Q}_{1} \omega_{1} \rho^{2}$ and $\omega_{1}=-V_{1}+0.5 v_{1}^{2}$. Similarly, the expectation of $Q_{t+1}+A_{t+1}$ conditional on being in the high risk state at $t+1$ is

$$
E_{t+1}\left(Q_{t+1}+A_{t+1} \mid t+1 \text { is high }\right)=a_{1, h i g h}+a_{2, h i g h} S_{t}+a_{3, h i g h} S_{t}^{2}
$$

where $a_{1, \text { high }}=\tilde{Q}_{2}\left(1+\omega_{2} \sigma^{2}\right)+\bar{A}, a_{2, h i g h}=\tilde{Q}_{2} v_{2} \rho+m \bar{A} \rho, a_{3, h i g h}=\tilde{Q}_{2} \omega_{2} \rho^{2}$ and $\omega_{2}=-V_{2}+0.5 v_{2}^{2}$.

The expectation of $Q_{t+1}+A_{t+1}$ is then

$$
\begin{aligned}
& E_{t}\left(Q_{t+1}+A_{t+1}\right) \\
= & p_{1} E_{t+1}\left(Q_{t+1}+A_{t+1} \mid t+1 \text { is low }\right)+\left(1-p_{1}\right) E_{t+1}\left(Q_{t+1}+A_{t+1} \mid t+1 \text { is high }\right) \\
= & d_{1, \text { low }}+d_{2, \text { low }} S_{t}+d_{3, \text { low }} S_{t}^{2}
\end{aligned}
$$


where $d_{i, \text { low }}=p_{1} a_{i, \text { low }}+\left(1-p_{1}\right) a_{i, h i g h}, i=1,2,3$.

The variance of $Q_{t+1}+A_{t+1}$ is

$$
\operatorname{var}\left(Q_{t+1}+A_{t+1}\right)=E_{t}\left(Q_{t+1}+A_{t+1}\right)^{2}-\left(E_{t}\left(Q_{t+1}+A_{t+1}\right)\right)^{2}
$$

Dropping terms in $S_{t}$ that are third and higher order, (77) gives

$$
\left(E_{t}\left(Q_{t+1}+A_{t+1}\right)\right)^{2}=d_{1, \text { low }}^{2}+2 d_{1, \text { low }} d_{2, \text { low }} S_{t}+\left(d_{2, \text { low }}^{2}+2 d_{1, \text { low }} d_{3, \text { low }}\right) S_{t}^{2}
$$

Next consider $E_{t}\left(Q_{t+1}+A_{t+1}\right)^{2}$. Conditional on being in a low risk state at $t+1$, we have

$$
Q_{t+1}+A_{t+1}=a_{1, \text { low }}+a_{2, l o w} S_{t}+a_{3, \text { low }} S_{t}^{2}+a_{4, \text { low }} \epsilon_{t+1}
$$

where $a_{4, \text { low }}=\tilde{Q}_{1}\left(v_{1}+\omega_{1} 2 \rho S_{t}\right)+m \bar{A}$. Using the definition of $a_{4, \text { low }}$, we then have

$$
E_{t}\left(\left(Q_{t+1}+A_{t+1}\right)^{2} \mid t+1 \text { is low }\right)=b_{1, \text { low }}+b_{2} S_{t, l o w}+b_{3} S_{t, l o w}^{2}
$$

where $b_{1, \text { low }}=a_{1, \text { low }}^{2}+\left(\tilde{Q}_{1} v_{1}+m \bar{A}\right)^{2} \sigma^{2}, b_{2, l o w}=2 a_{1, \text { low }} a_{2, l o w}+4 \tilde{Q}_{1}\left(\tilde{Q}_{1} v_{1}+m \bar{A}\right) \omega_{1} \rho \sigma^{2}$, and $b_{3, \text { low }}=a_{2, \text { low }}^{2}+2 a_{1, \text { low }} a_{3, \text { low }}+4 \tilde{Q}_{1}^{2} \omega_{1}^{2} \rho^{2} \sigma^{2}$. Similarly, conditional on being in a high risk state at $t+1$ we have

$$
E_{t}\left(\left(Q_{t+1}+A_{t+1}\right)^{2} \mid t+1 \text { is high }\right)=b_{1, h i g h}+b_{2, h i g h} S_{t}+b_{3, h i g h} S_{t}^{2}
$$

Here $b_{i, h i g h}(i=1,2,3)$ is defined analogously to $b_{i, l o w}$ with subscripts low replaced by high and subscripts 1 for $\tilde{Q}, v$ and $\omega$ replaced by 2 . This implies that in the low risk state at $t$ :

$$
E_{t}\left(Q_{t+1}+A_{t+1}\right)^{2}=c_{1, l o w}+c_{2, l o w} S_{t}+c_{3, l o w} S_{t}^{2}
$$

where $c_{i, \text { low }}=p_{1} b_{i, \text { low }}+\left(1-p_{1}\right) b_{i, h i g h}, i=1,2,3$.

It follows that

$$
\begin{aligned}
& \operatorname{var}\left(Q_{t+1}+A_{t+1}\right)=\left(c_{1, \text { low }}-d_{1, \text { low }}^{2}\right)+\left(c_{2, \text { low }}-2 d_{1, \text { low }} d_{2, \text { low }}\right) S_{t} \\
& +\left(c_{3, \text { low }}-d_{2, \text { low }}^{2}-2 d_{1, \text { low }} d_{3, \text { low }}\right) S_{t}^{2}
\end{aligned}
$$

Finally, a quadratic approximation around $S_{t}=0$ of $Q_{t} R_{t+1}$ gives

$$
Q_{t} R_{t+1}=e_{1, \text { low }}+e_{2, \text { low }} S_{t}+e_{3, \text { low }} S_{t}^{2}
$$


where $e_{1, l o w}=\frac{1}{\eta}\left[(\nu-W)+K \tilde{Q}_{1}\right] \tilde{Q}_{1}, e_{2, l o w}=\frac{1}{\eta}\left[(\nu-W)+2 K \tilde{Q}_{1}\right] \tilde{Q}_{1} v_{1}$ and $e_{3, \text { low }}=\frac{1}{\eta}\left[(\nu-W) \omega_{1}+2 K \tilde{Q}_{1}\left(-V_{1}+v_{1}^{2}\right)\right] \tilde{Q}_{1}$.

Substituting these results into the market equilibrium condition (27), and taking a second order approximation around $S_{t}=0$, again gives (29). Setting $Z_{0}=0$, $Z_{1}=0$ and $Z_{2}=0$ gives respectively

$$
\begin{aligned}
& \bar{W}\left(d_{1, \text { low }}-e_{1, \text { low }}\right)=K\left(c_{1, \text { low }}-d_{1, \text { low }}^{2}\right) \\
& \bar{W}\left(d_{2, \text { low }}-e_{2, \text { low }}\right)=K\left(c_{2, \text { low }}-2 d_{1, \text { low }} d_{2, \text { low }}\right) \\
& \bar{W}\left(d_{3, \text { low }}-e_{3, \text { low }}\right)=K\left(c_{3, \text { low }}-d_{2, \text { low }}^{2}-2 d_{1, \text { low }} d_{3, \text { low }}\right)
\end{aligned}
$$

All of this is conditional on being in the low risk state at $t$. We can similarly impose market equilibrium conditional on being in the high risk state at $t$. Define $c_{i, h i g h}$ and $d_{i, h i g h}(i=1,2,3)$ the same as $c_{i, \text { low }}$ and $d_{i, \text { low }}$, with $p_{1}$ replaced by $1-p_{2}$. Also define $e_{i, \text { high }}(i=1,2,3)$ the same as $e_{i, l o w}$, with the subscripts 1 for $\tilde{Q}, v, V$ and $\omega$ replaced by subscripts 2 . Then imposing market clearing we get three equations analogous to (86)-(88) with the subscripts low replaced by high. Solving these six equations jointly gives the solutions for $\tilde{Q}_{1}, \tilde{Q}_{2}, v_{1}, v_{2}, V_{1}$ and $V_{2}$. This is done numerically in Gauss, using as starting values the solutions for equilibria 1 and 2 without switching. 


\section{References}

[1] Acharya, Viral V. and Lasse H. Pedersen (2005), "Asset Pricing and Liquidity Risk," Journal of Financial Economics 77(2), 375-410.

[2] Adrian, Tobias and Hyun S. Shin (2008), "Financial Intermediary Leverage and Value-at-Risk," Federal Reserve Bank of New York Staff Reports no. 338.

[3] Allen, Franklin and Douglas Gale (1994), "Limited Market Participation and Volatility of Asset Prices," American Economic Review 84, 933-955.

[4] Amihud, Yakov, Haim Mendelson and Lasse H. Pedersen (2005), Liquidity and Asset Prices, Foundations and Trends in Finance, now Publishers.

[5] Bacchetta, Philippe and Eric van Wincoop (2006), "Can Information Heterogeneity Explain the Exchange Rate Determination Puzzle?," American Economic Review 96, 552-576.

[6] Bacchetta, Philippe and Eric van Wincoop (2010), "On the Global Spread of Risk Panics," mimeo.

[7] Bacchetta, Philippe, Cédric Tille, and Eric van Wincoop (2010), "Leverage and Risk Panics," mimeo.

[8] Barro, Robert J. (2006), "Rare Disasters and Asset Markets in the Twentieth Century," Quarterly Journal of Economics 121(3), 823-866.

[9] Bloom, Nicholas (2009), "The Impact of Uncertainty Shocks," Econometrica 77, 623-685.

[10] Brunnermeier, Markus K. and Lasse H. Pedersen (2009), "Market Liquidity and Funding Liquidity," Review of Financial Studies 22, 2201-2238.

[11] Brunnermeier, Markus K. and Yuliy Sannikov (2009), "A Macroeconomic Model with a Financial Sector," mimeo.

[12] Caballero, Ricardo and Arvind Krishnamurthy (2008), "Collective Risk Management in a Flight to Quality Episode," Journal of Finance LXIII (5), 21952230 . 
[13] Campell, Rachel, Ronald Huisman, and Kees Koedijk (2001), "Optimal Portfolio Selection in a Value-at-Risk framework," Journal of Banking and Finance 25, 1789-1804.

[14] Danielsson, J., H.S. Shin, and J.-P. Zigrand (2009), "Risk Appetite and Endogenous Risk," mimeo.

[15] Fostel, Ana and John Geanakoplos (2008), "Leverage Cycles and the Anxious Economy," American Economic Review 98(4), 1211-1244.

[16] Gabaix, Xavier (2009), "Variable Rare Disasters: An Exactly Solved Framework for Ten Puzzles in Macro-Finance," mimeo.

[17] Goyenko, Ruslan, Craig Holden and Charles Trzcinka (2009), "Do Liquidity Measures Measure Liquidity?," Journal of Financial Economics 92, 153-181.

[18] Gromb, Denis and Dimitri Vayanos (2002), "Equilibrium and Welfare in Markets with Financially Constrained Arbitrageurs," Journal of Financial Economics 66, 361-407.

[19] Gromb, Denis and Dimitri Vayanos (2008), "Leverage and Liquidity Dry-Ups: A Framework and Policy Implications," mimeo.

[20] He, Zhiguo. and Arvind Krishnamurthy (2008a), "Intermediary Asset Pricing," NBER WP No. 14517.

[21] He, Zhiguo and Arvind Krishnamurthy (2008b), "A Model of Capital and Crises," NBER WP No. 14366.

[22] Jeanne, Olivier and Andrew K. Rose (2002), "Noise Trading and Exchange Rate Regimes," Quarterly Journal of Economics 77, 537-569.

[23] Kyle, Albert S. and Wei Xiong (2001), "Contagion as a Wealth Effect," The Journal of Finance 56(4), 1401-1440.

[24] Manuelli, Rodolfo E. and James Peck (1992), "Sunspot-like Effects of Random Endowments," Journal of Economic Dynamics and Control 16, 193-206. 
[25] McCafferty, Stephen and Robert Driskill (1980), "Problems of Existence and Uniqueness in Nonlinear Rational Expectations Models," Econometrica 48, 1313-1317.

[26] Pagano, Marco (1989), "Endogenous Market Thinness and Stock Price Volatility," Review of Economic Studies 56, 269-288.

[27] Spear, Stephen E., Sanjay Srivastava, and Michael Woodford (1990), "Indeterminacy of Stationary Equilibrium in Stochastic Overlapping Generations Models," Journal of Economic Theory 50, 265-84.

[28] Vayanos, Dimitri and Jiang Wang (2009), "Liquidity and Asset Prices: A Unified Framework" CEPR Discussion Paper No. 7410.

[29] Xiong, Wei (2001), "Convergence Trading with Wealth Effects: An Amplification Mechanism in Financial Markets," Journal of Financial Economics 62, 247-292. 


\section{Figure 1 Sunspot Equilibria*}

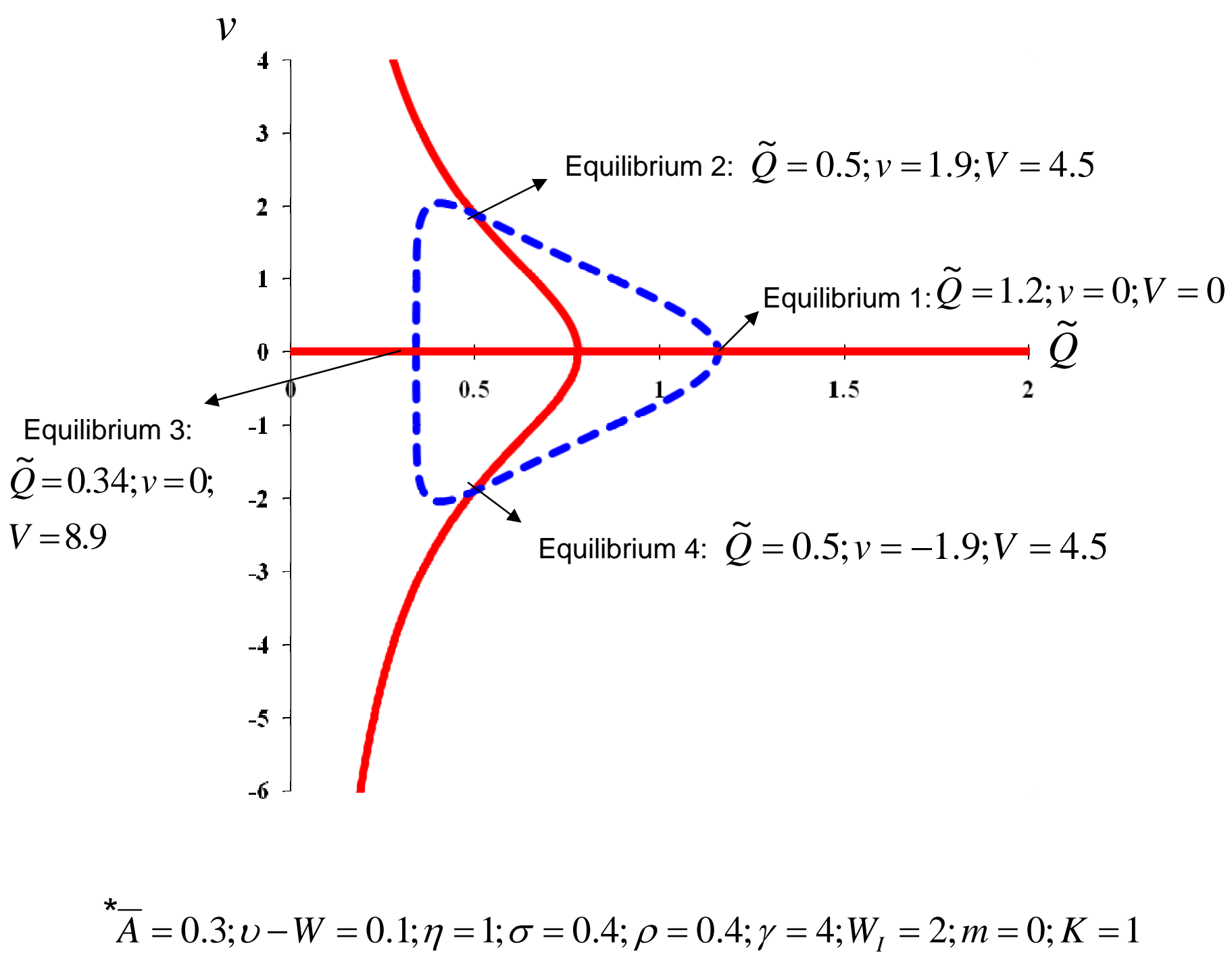




\section{Figure 2 Sunspot-Like Equilibria*}

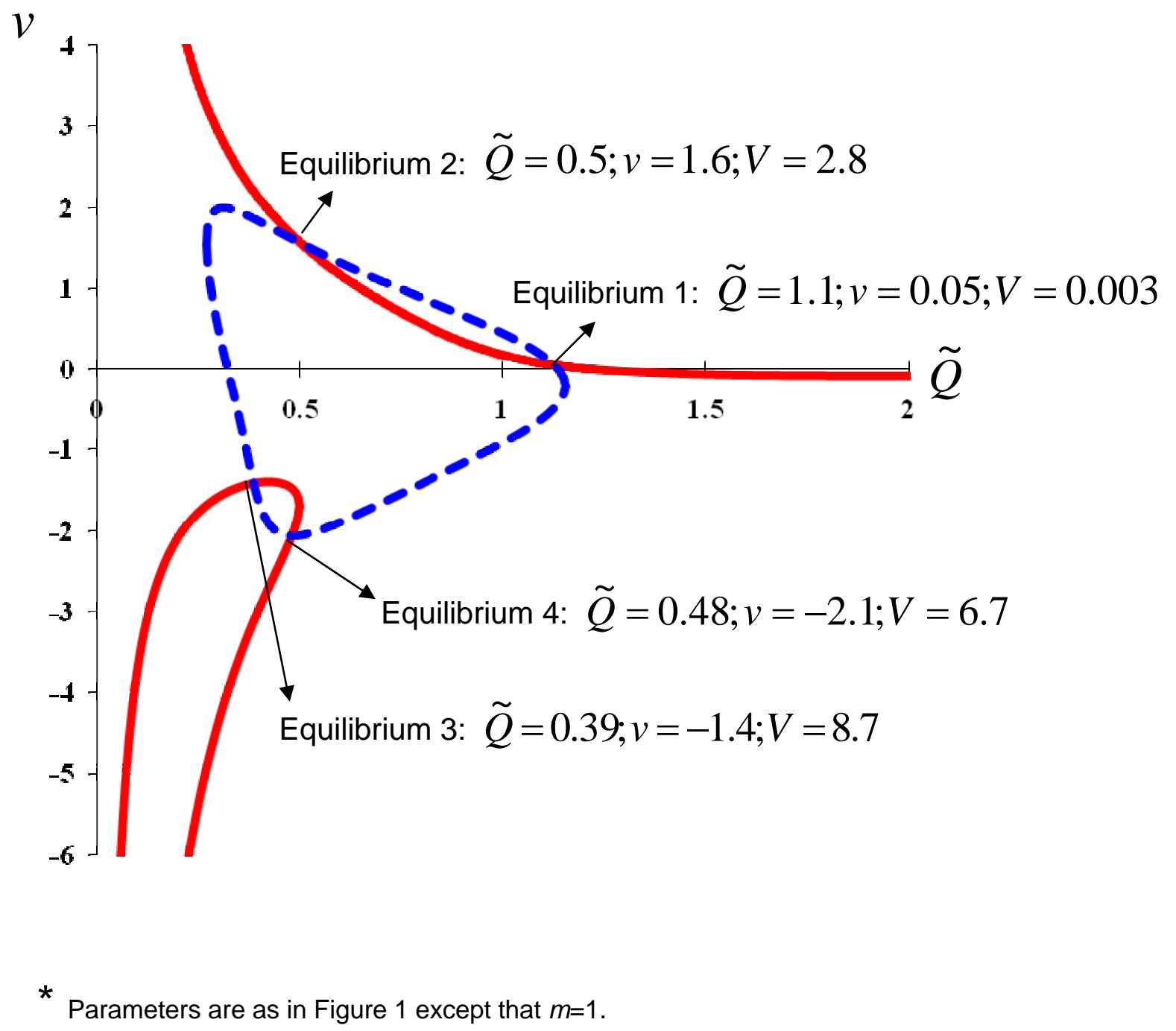


Figure 3 Solution as Function of $m$ (Equilibrium 2)*
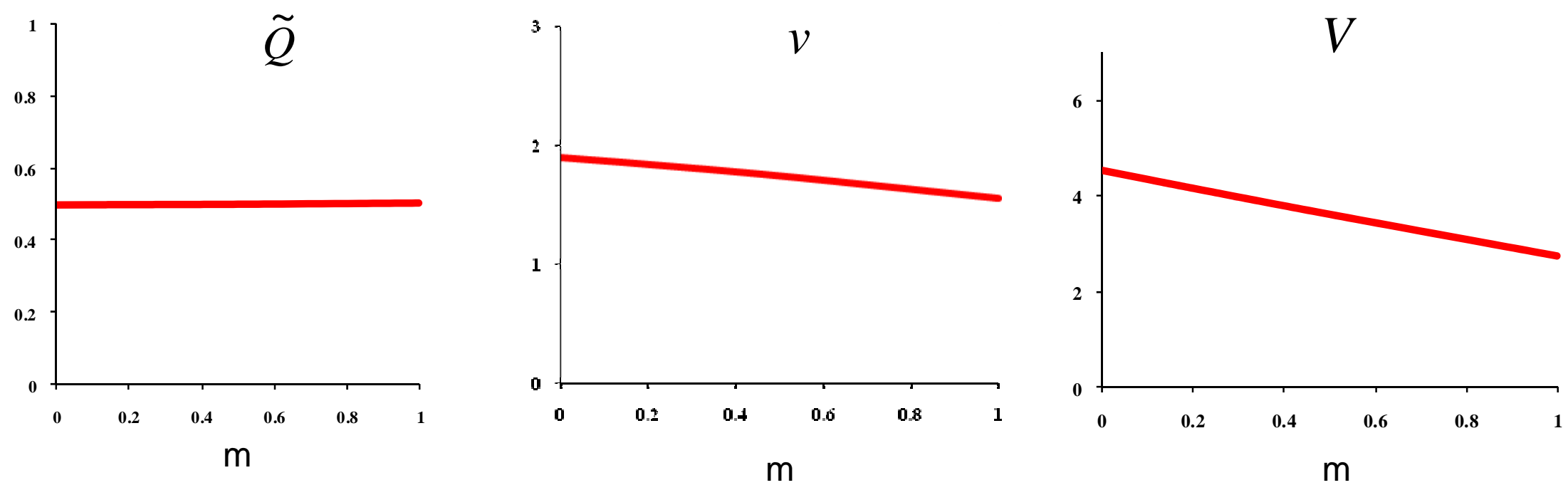

* Parameters are identical to those in Figure 1. 


\section{Figure 4 Switching Equilibria*}

\section{solid=low risk state; broken=high risk state}
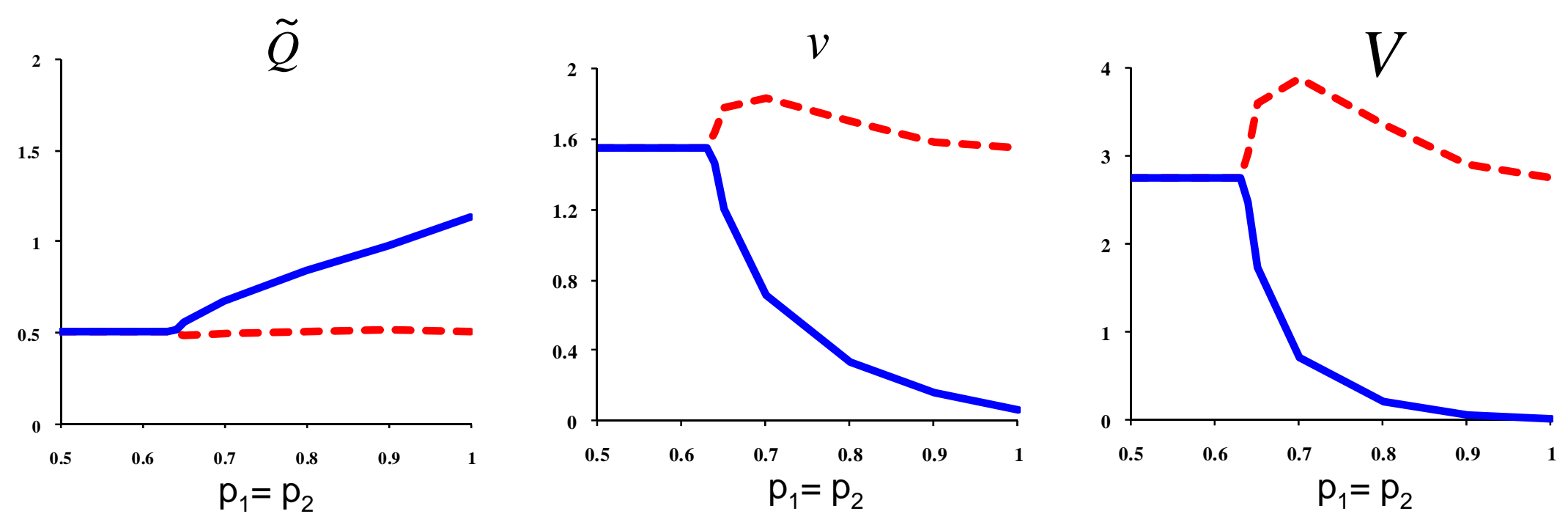

* This is based on the parameters of Figure 2. When $p_{1}=p_{2}=1$, the high and low risk states correspond exactly to equilibria 1 and 2 in Figure 2. 
Figure 5 Equilibria with Financial Shocks: No Persistence*

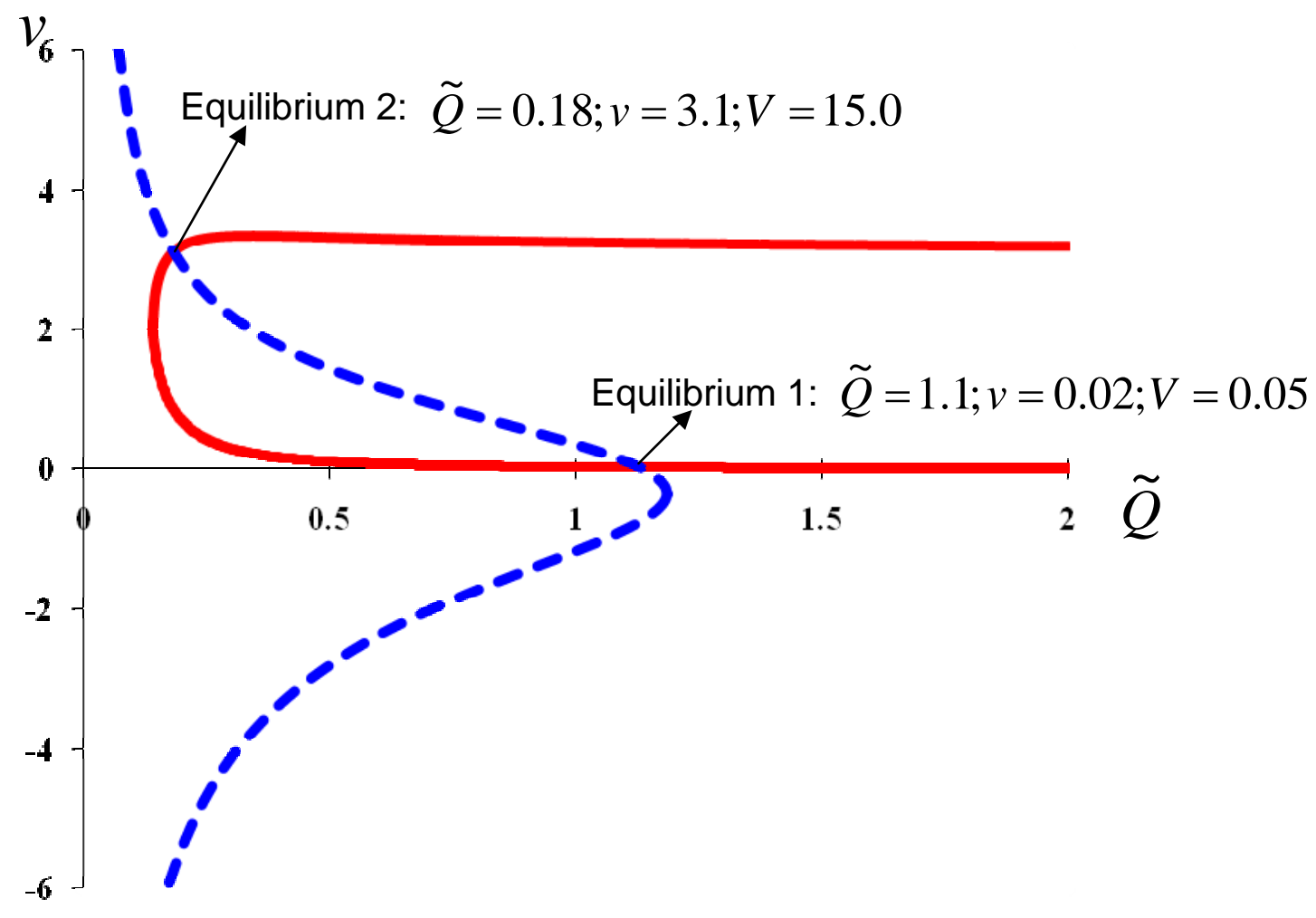

* $\bar{A}=0.3 ; v-W=0.1 ; \eta=1 ; \sigma=0.4 ; \sigma_{\theta}=0.4 ; \rho_{\theta}=0 ; \gamma=4 ; \bar{W}_{I}=2 ; m=2 ; K=1$ 


\section{Figure 6 Sunspot and Sunspot-Like Equilibria with Financial Shocks*}

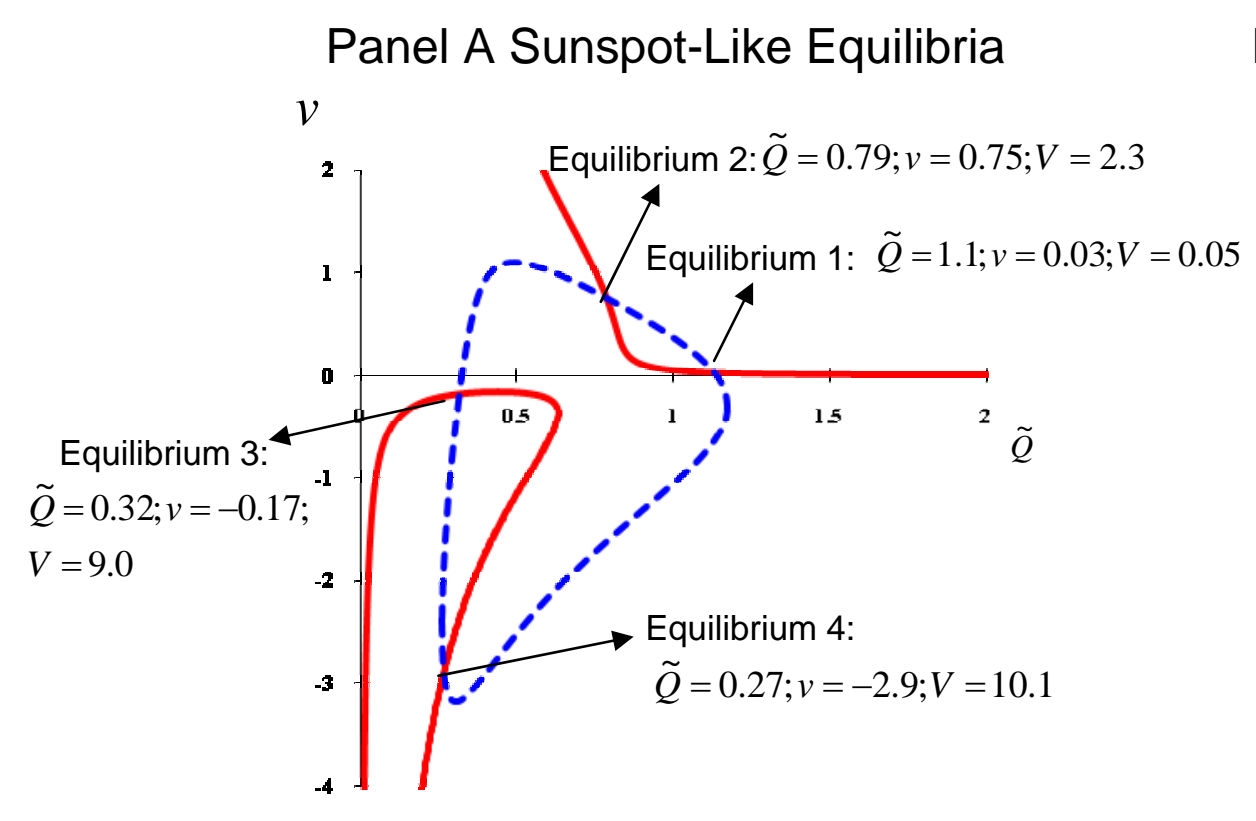

\section{Panel B Sunspot Equilibria}

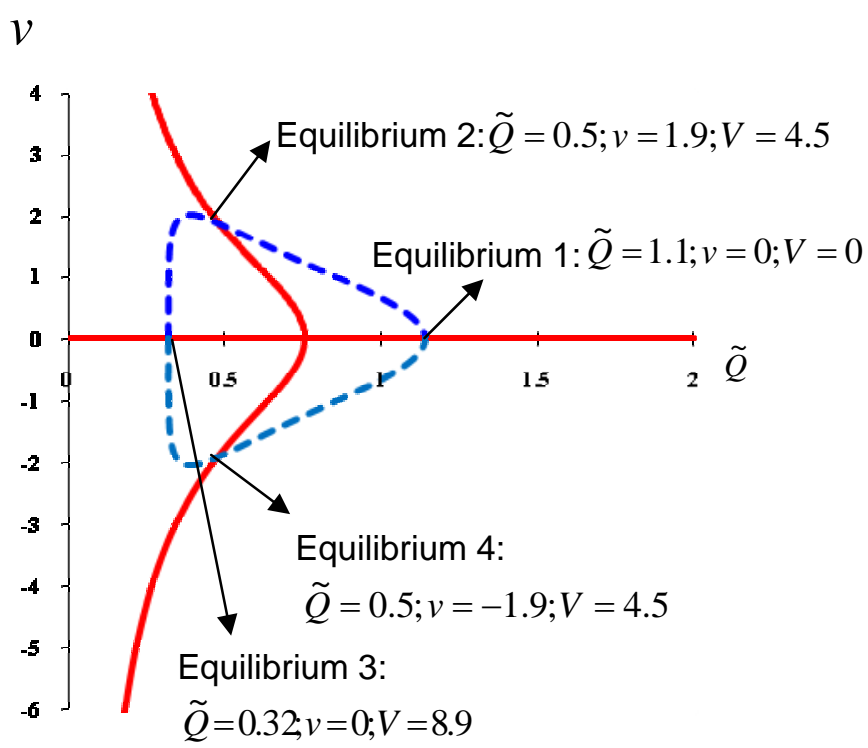

\footnotetext{
* Panel A assumes $\rho_{\theta}=0.4, m=2$. Panel B assumes $\rho_{\theta}=0.4, m=0$; otherwise the parameters are the same as in Figure 5.
} 


\section{Figure 7b Net worth, leverage and illiquidity}

\section{Net worth brokers and dealers}

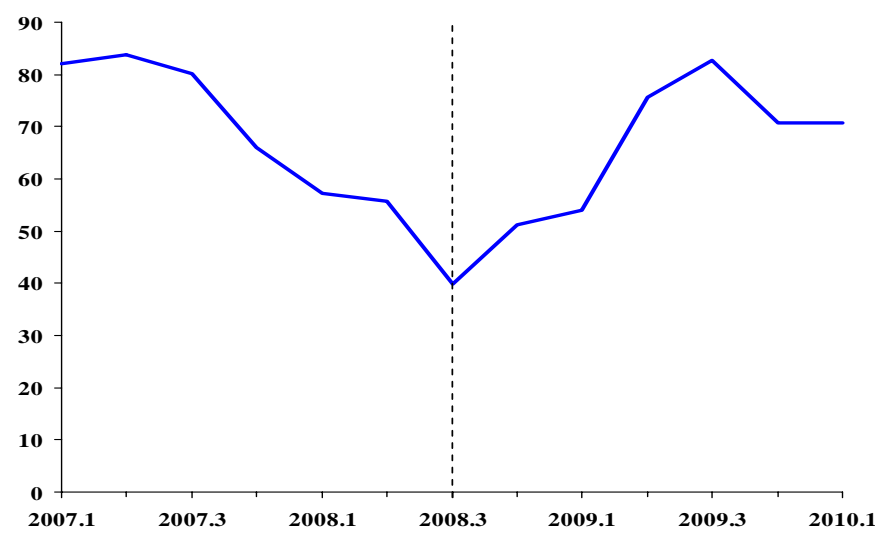

\section{Leverage brokers and dealers}

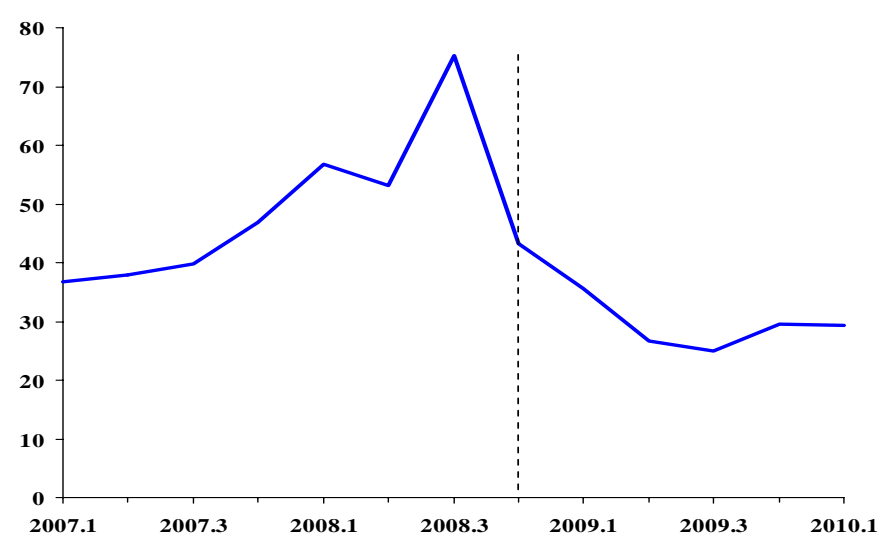

illiquidity

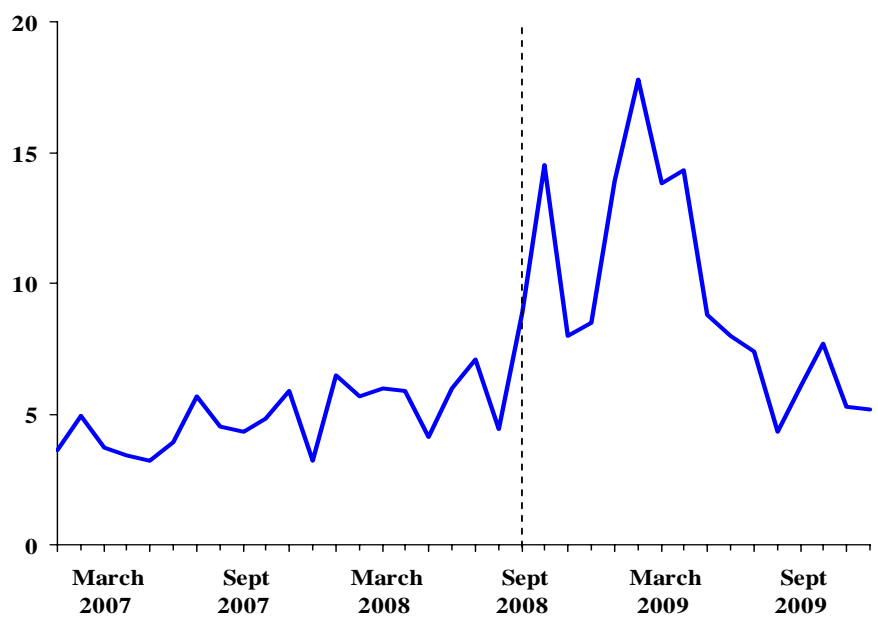

Source: Data on brokers and dealers from the Fed's Flow of Funds (L.129); net worth is assets minus liabilities, billion US \$; leverage is net worth divided by assets. The illiquidity measure is an updated measure of Amihud (2002). The vertical lines represent Q3 2008 for the quarterly net worth and leverage series and September 2008 for the monthly illiquidity series. 


\section{Figure 8 Model Simulation*}

shaded area $=$ high risk equilibrium; vertical lines $=$ endowment shock

Wealth of investors

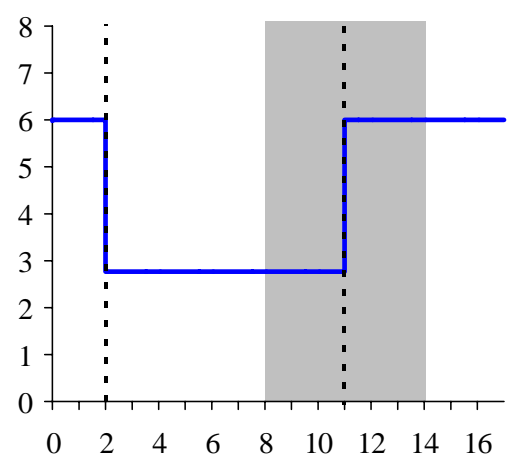

Gross Interest Rate

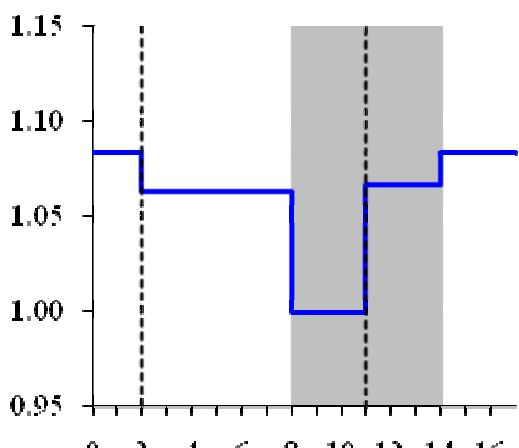

Equity Price

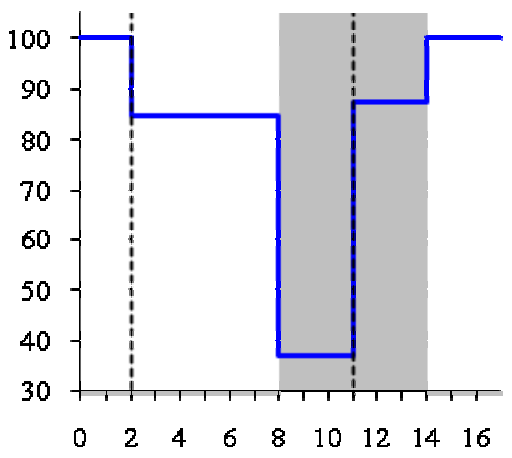

Leverage

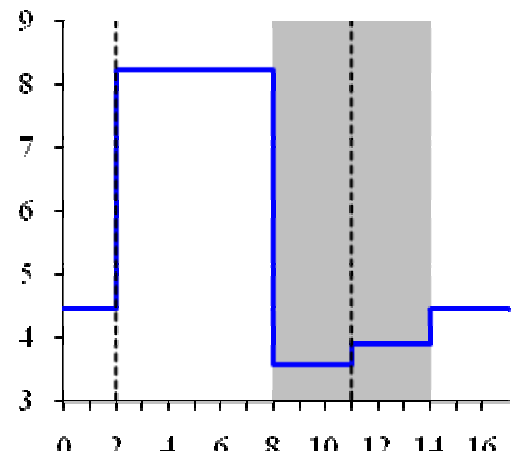

Risk Equity Price

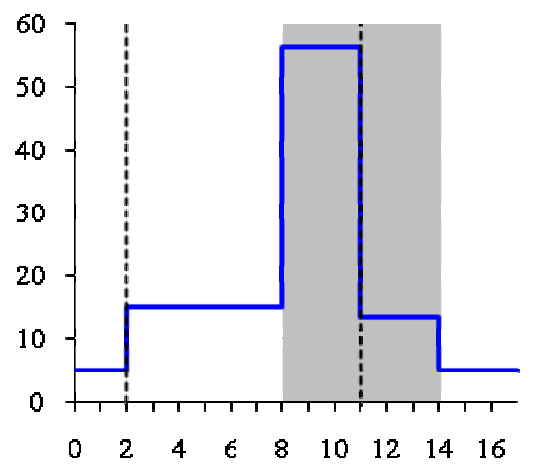

illiquidity

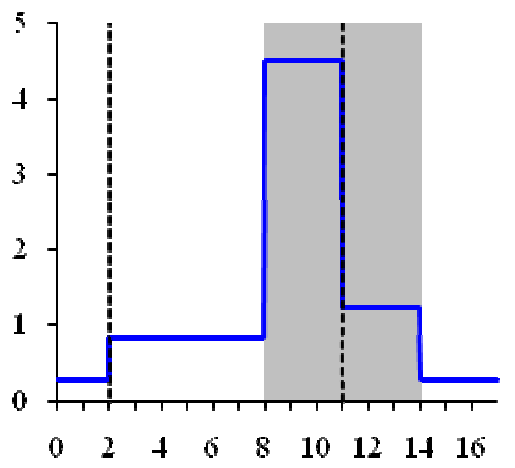

* The economy starts in the low risk equilibrium. At time 2 the endowment of investors falls from 6 to 2.8 . The economy stays in the low risk equilibrium until time 8, at which point is shifts to the high risk equilibrium. At time 11 endowments shift back towards the initial allocation. The economy remains in the high risk equilibrium until time 14 , at which points it shifts back to the low risk equilibrium.

$\bar{A}=0.15 ; v-W=190 ; \eta=200 ; \sigma_{a}=0.1 ; \sigma_{\theta}=0.1 ; \rho_{\theta}=0.7 ; \gamma=1 ; \bar{W}_{I}=6 ; m=2 ; K=20 ; p_{1}=0.95 ; p_{2}=0.7$ 